\title{
Networked Fault-Tolerant Control Allocation for Multiple Actuator Failures
}

\author{
Inseok Yang ${ }^{1}$ and Dongik Lee ${ }^{2}$ \\ ${ }^{1}$ Center for ICT \& Automobile Convergence, Kyungpook National University, Daegu 702-701, Republic of Korea \\ ${ }^{2}$ School of Electronics Engineering, Kyungpook National University, Daegu 702-701, Republic of Korea \\ Correspondence should be addressed to Dongik Lee; dilee@ee.knu.ac.kr
}

Received 3 May 2014; Revised 8 August 2014; Accepted 24 August 2014

Academic Editor: Qingsong Xu

Copyright (C) 2015 I. Yang and D. Lee. This is an open access article distributed under the Creative Commons Attribution License, which permits unrestricted use, distribution, and reproduction in any medium, provided the original work is properly cited.

This paper proposes intelligent fault-tolerant control technique using network. Not only control commands generated by a controller but also diagnostic data for tolerating failures can be transmitted through network. In this paper, fault-tolerant control allocation method (FTCA) is proposed to tolerate failures in more than one actuator. FTCA is based on a well-known actuator management technique called control allocation (CA). While the conventional CA is used to redistribute actuators optimally, FTCA redistributes actuators to compensate for the performance degradation due to actuator failure. To analyze the effects of faulty actuator, this paper proposes the general model of the faulty system firstly. And then the modified CA for tolerating the effect of failure is proposed. The performance of the proposed FTCA method is verified by the numerical simulations with application to F-18 High Alpha Research Vehicle (HARV).

\section{Introduction}

Systems, such as aircrafts, spacecrafts, and automotive vehicles, that lead to a catastrophic accident due to failures are called "safety-critical systems." Since these systems require high-level of dependability, redundant actuators are usually adopted. Redundant actuators are considered as back-up systems that activate as main actuators after occurring failure in the primary ones. However, adopting redundant actuators takes disadvantages of fuel-, space-, cost-, and weightinefficiency during normal operation. In contrast to adopting redundant actuators, techniques based on control theory have been proposed for last 30 years: for example, pseudoinverse [1], model reference adaptive control [2], sliding mode control [3], multiple model switching and tuning [4], control allocation [5-10], and so forth. The goal of these methods is to provide the feasible control input for a faulty system by reconfiguring a controller. Hence, these methods are defined as fault-tolerant control (FTC) and also called reconfiguration. FTC techniques can be classified into three categories: pole-assignment, multiple model, and allocating actuators.
First, pole-assignment method is to move the pole position from the undesired position due to faulty system dynamics to the position that can keep the normal performance. In a broad sense, pseudoinverse, model reference adaptive control, and sliding mode control are comprised in this method. To design pole-assignment fault-tolerant controller, the stability of the closed-loop control system according to actuator operation range must be considered. Second, in multiple model method, reconfiguration can be achieved by selecting a feasible controller with respect to the faulty system model. So a group of possible faulty system models and their controllers are predesigned. Once failure occurs, multiple model fault-tolerant controller estimates the closest faulty model in the group and then selects the corresponding controller. The main disadvantage of this method is that the number of possible models is increased exponentially in order to tolerate all possible failures. Control allocation is an actuator management technique in overactuated system that has larger number of actuators than the number of states. For normal operation, redundant actuators are used to improve the performance of the system. For example, thrust 


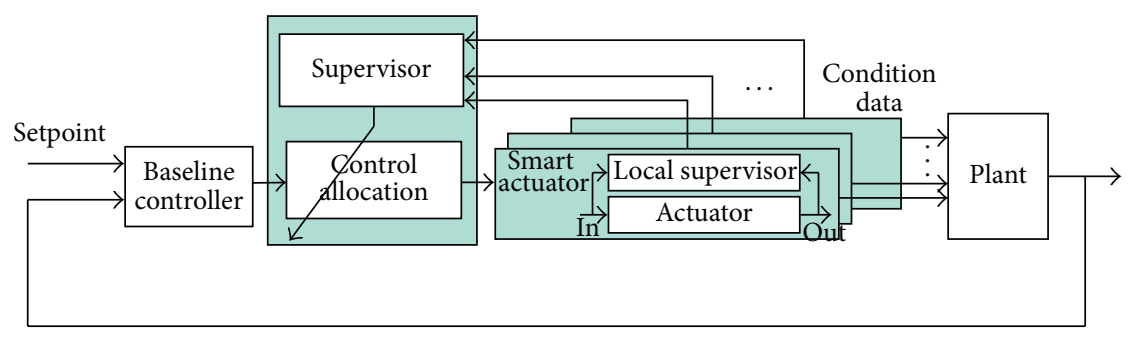

FIGURE 1: The proposed networked fault-tolerant control system [9].

vectoring nozzles are adopted as redundant actuators to achieve high angle-of-attack flight ability in modern aircrafts. These redundant actuators can also provide an opportunity to compensate the effect of failure. This paper modifies the conventional CA to achieve the fault-tolerant ability for failures on several actuators.

However, reconfiguration methods shown above must require fast and accurate failure information to tolerate failure. For this reason, fault detection and diagnosis (FDD) technique is generally introduced [11]. Ideally, failure can be compensated by FTC using the diagnostic data generated by FDD. However, most of FDD methods have been developed as monitoring/alarm systems in order to achieve safety by providing accurate failure information to users. Although FDD diagnoses health condition accurately, there are some differences between the diagnostic data generated by FDD and the required data for tolerating failure by FTC. These differences make it hard to apply the diagnostic data for faulttoleration [11].

Recent advances in network communication provide opportunities to transmit failure-information data among controllers, actuators, and sensors by interconnecting the control nodes through fieldbus network such as CAN and FlexRay $[9,10,12,13]$. The control system interconnected by network is defined as networked control system (NCS). NCS provides not only the advantages of simplified wiring but also the opportunity to implement more sophisticated control laws such as FTC. Two major research issues have been studied in applying NCS to a real system: one is the stability problem that occurred in network environments such as transmission delay and packet losses among network nodes and the other is to design a sophisticated control architecture including FTC. Actually, the stability problems due to message delay or packet loss can be resolved by adopting a highly reliable and deterministic network such as FlexRay and TTP/C. Recently, more researchers have paid attention to the second issue. Thanks to the enhanced dataexchange ability, NCS can be efficiently used to tolerate failures by exchanging the diagnostic data fast and accurately. For example, Yang et al. proposed the networked faulttolerant control system (NFTCS) architecture using smart actuators [9]. In Figure 1, by adopting microprocesses on conventional actuators, smart actuators can achieve the selfdiagnostic and communication abilities for sharing their health conditions. Then the supervisor can analyze the faulty system model and reconfigure the baseline controller that guarantees the stability of the faulty system using the shared health condition data. In this paper, the FTCA method using the transmitted condition data through network is proposed.

This paper is organized as follows. Section 2 analyzes the general dynamic model of the actuator faulty system. Then fault-tolerant control allocation method is proposed in Section 3. In this section, actuator saturation problem due to limited operation range of actuators is also considered. In Section 4, the performance of the proposed FTCA method is evaluated by simulation results with application to the F-18 HARV aircraft. Finally, the concluding remarks followed in Section 5.

\section{General Dynamic Model of Actuator Faulty System}

In this section, the general dynamic model of an actuator failure system is analyzed. The condition data provided by smart actuators are used to construct the mathematical model of the faulty system. First, the generalized model of a faulty actuator is proposed. And then it is expanded to the set of several faulty actuators. Finally, the general state-space form of the actuator failure system is proposed at the end of this section.

2.1. Generalized Actuator Failure Model. Bošković and Mehra proposed that the response of faulty actuator can be represented as one of the following four types: lock-in-place (LiP), hardover, float, and loss of effectiveness (LoE) [14]. Figure 2 shows the actuator responses of normal and failure cases. In Figure 2, failure responses are categorized into two types according to the input-output relationship; that is, one is unstable case without any consideration of input commands since faulty actuator stops at one position or diverges to the saturation position like LiP, hardover, and float failures and the other is performance degradation case relative to input commands. In [9], the former one is defined as total failure.

From Figure 2, the general position of the $i$ th actuator with/without failures can be represented as follows:

$$
u_{i}=\llbracket \gamma_{i} \rrbracket \gamma_{i} u_{\text {normal }, i}+\left(1-\llbracket \gamma_{i} \rrbracket\right) \bar{u}_{i}
$$

where the subscript $i$ indicates the $i$ th actuator and $\llbracket \cdot \rrbracket$ is the smallest integer greater than or equal to $\cdot$. And $u_{\text {normal }, i}$ denotes the expected position of the $i$ th actuator if it is normal and $\bar{u}_{i}$ denotes the total failure position if total failure occurs. 


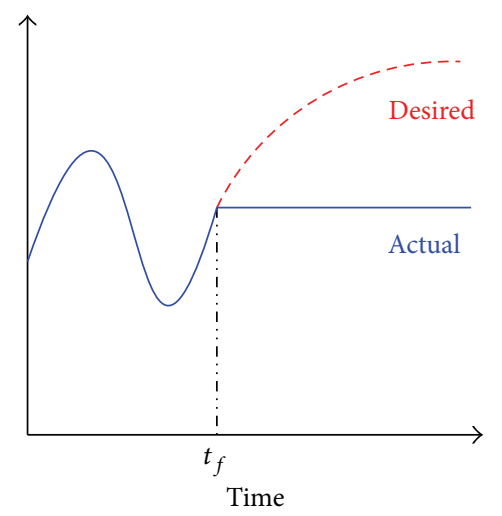

(a) Lock-in-place

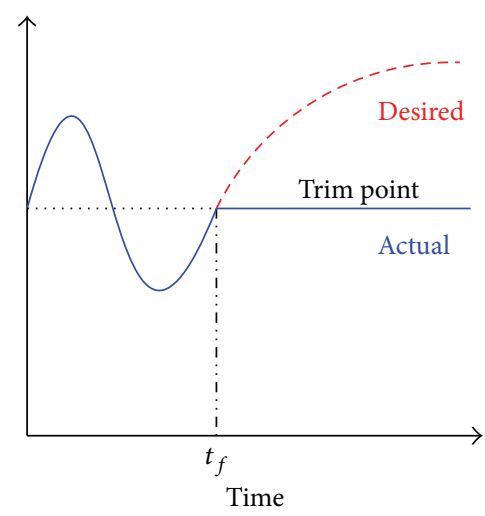

(b) Float

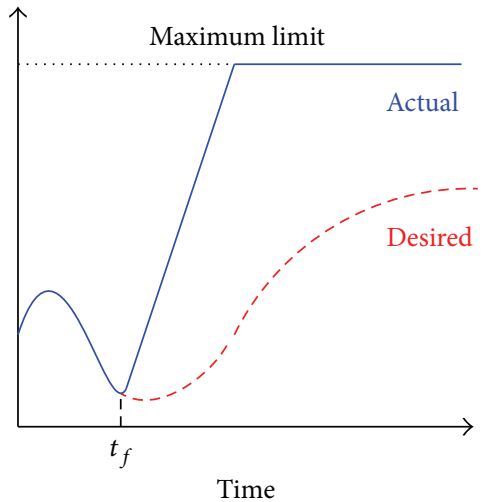

(c) Lock-in-place

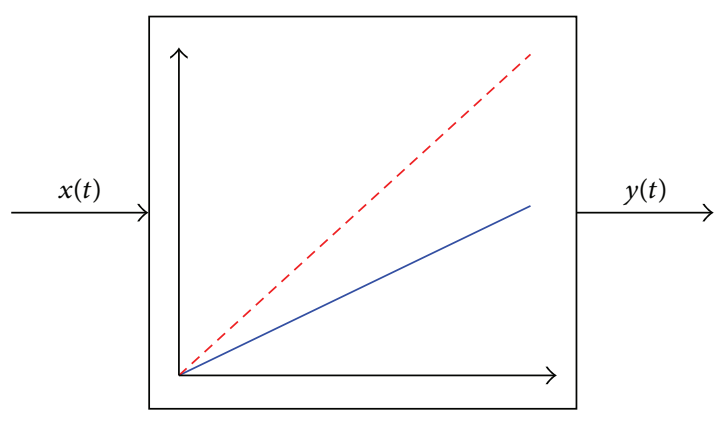

(d) Float

FIgURE 2: Typical failures of actuator [14].

The quantitative value $\gamma_{i}$ in the range of 0 to 1 indicates the performance degradation of the $i$ th actuator:

$$
\gamma_{i}= \begin{cases}0, & \text { if total failure occurs } \\ \varepsilon(0<\varepsilon<1), & \text { if LoE failure occurs } \\ 1, & \text { if the surface is normal. }\end{cases}
$$

The quantitative value $\gamma_{i}$ is provided by the $i$ th smart actuator as the condition data; that is, if $50 \%$ of LoE failure occurs on the $i$ th actuator, then $\gamma_{i}=0.5$; so $u_{i}=0.5 u_{\text {normal, } i}$. Similarly, if the $i$ th actuator is operated normally, then $\gamma_{i}=1$; so $\delta_{i}=u_{i}$.

Let $m$ be total number of actuators. If failures occurred on $k(1 \leq k \leq m)$ actuators, then the general dynamics of a group of faulty actuators can be represented as follows:

$$
\begin{aligned}
{\left[\begin{array}{c}
u_{1} \\
u_{2} \\
\vdots \\
u_{k}
\end{array}\right]=} & {\left[\begin{array}{cccc}
\llbracket \gamma_{1} \rrbracket \gamma_{1} & 0 & \cdots & 0 \\
0 & \llbracket \gamma_{2} \rrbracket \gamma_{2} & \cdots & 0 \\
\vdots & \vdots & \ddots & \vdots \\
0 & 0 & \cdots & \llbracket \gamma_{k} \rrbracket \gamma_{k}
\end{array}\right]\left[\begin{array}{c}
u_{\text {normal }, 1} \\
u_{\text {normal }, 2} \\
\vdots \\
u_{\text {normal }, k}
\end{array}\right] } \\
+ & {\left[\begin{array}{ccccc}
1-\llbracket \gamma_{1} \rrbracket & 0 & \cdots & 0 \\
0 & 1-\llbracket \gamma_{2} \rrbracket & \cdots & 0 \\
\vdots & \vdots & \ddots & \vdots \\
0 & 0 & \cdots & 1-\llbracket \gamma_{k} \rrbracket
\end{array}\right]\left[\begin{array}{c}
\bar{u}_{1} \\
\bar{u}_{2} \\
\vdots \\
\bar{u}_{k}
\end{array}\right] }
\end{aligned}
$$

$$
\begin{gathered}
=\left[\begin{array}{cccc}
\llbracket \gamma_{1} \rrbracket & 0 & \cdots & 0 \\
0 & \llbracket \gamma_{2} \rrbracket & \cdots & 0 \\
\vdots & \vdots & \ddots & \vdots \\
0 & 0 & \cdots & \llbracket \gamma_{k} \rrbracket
\end{array}\right]\left[\begin{array}{cccc}
\gamma_{1} & 0 & \cdots & 0 \\
0 & \gamma_{2} & \cdots & 0 \\
\vdots & \vdots & \ddots & \vdots \\
0 & 0 & \cdots & \gamma_{k}
\end{array}\right] \\
\times\left[\begin{array}{c}
u_{\text {normal }, 1} \\
u_{\text {normal }, 2} \\
\vdots \\
u_{\text {normal }, k}
\end{array}\right]
\end{gathered}
$$$$
+\left\{\left[\begin{array}{cccc}
1 & 0 & \cdots & 0 \\
0 & 1 & \cdots & 0 \\
\vdots & \vdots & \ddots & \vdots \\
0 & 0 & \cdots & 1
\end{array}\right]-\left[\begin{array}{cccc}
\llbracket \gamma_{1} \rrbracket & 0 & \cdots & 0 \\
0 & \llbracket \gamma_{2} \rrbracket & \cdots & 0 \\
\vdots & \vdots & \ddots & \vdots \\
0 & 0 & \cdots & \llbracket \gamma_{k} \rrbracket
\end{array}\right]\right\}
$$$$
\times\left[\begin{array}{c}
\bar{u}_{1} \\
\bar{u}_{2} \\
\vdots \\
\bar{u}_{k}
\end{array}\right]
$$$$
\underset{\text { Let }}{\Longrightarrow} \mathbf{u}_{F}=\llbracket \boldsymbol{\Gamma}_{F} \rrbracket \boldsymbol{\Gamma}_{F} \mathbf{u}_{\text {normal }, F}+\left(\mathbf{I}_{k}-\llbracket \boldsymbol{\Gamma}_{F} \rrbracket\right) \overline{\mathbf{u}}_{F},
$$ 
where $\mathbf{u}_{\text {normal, } F}$ denotes the set of expected normal outputs of faulty actuators and $\overline{\mathbf{u}}_{F}$ denotes the total failure positions. And $\mathbf{I}_{k}$ is an $(k \times k)$-identity matrix. Moreover,

$$
\begin{gathered}
\boldsymbol{\Gamma}_{F}=\left[\begin{array}{cccc}
\gamma_{1} & 0 & \cdots & 0 \\
0 & \gamma_{2} & \cdots & 0 \\
\vdots & \vdots & \ddots & \vdots \\
0 & 0 & \cdots & \gamma_{k}
\end{array}\right], \\
\llbracket \Gamma_{F} \rrbracket=\left[\begin{array}{cccc}
\llbracket \gamma_{1} \rrbracket & 0 & \cdots & 0 \\
0 & \llbracket \gamma_{2} \rrbracket & \cdots & 0 \\
\vdots & \vdots & \ddots & \vdots \\
0 & 0 & \cdots & \llbracket \gamma_{k} \rrbracket
\end{array}\right] .
\end{gathered}
$$

Let us denote the responses of the augmented actuators whose entries are sets of faulty actuators $\mathbf{u}_{F} \in \mathbf{R}^{k}$ and normal actuators $\mathbf{u}_{N} \in \mathbf{R}^{m-k}$ by $\mathbf{u}=\left[\mathbf{u}_{F}^{T}, \mathbf{u}_{N}^{T}\right]^{T}$; then the general responses of actuators can be represented as follows:

$$
\begin{aligned}
& {\left[\begin{array}{c}
\mathbf{u}_{F} \\
\mathbf{u}_{N}
\end{array}\right]=} {\left[\begin{array}{cc}
\llbracket \boldsymbol{\Gamma}_{F} \rrbracket & \mathbf{0}_{k \times(m-k)} \\
\mathbf{0}_{(m-k) \times k} & \llbracket \mathbf{I}_{m-k}
\end{array}\right]\left[\begin{array}{cc}
\boldsymbol{\Gamma}_{F} & \mathbf{0}_{k \times(m-k)} \\
\mathbf{0}_{(m-k) \times k} & \mathbf{I}_{m-k}
\end{array}\right] } \\
& \times\left[\begin{array}{c}
\mathbf{u}_{\text {normal }, F} \\
\mathbf{u}_{\text {normal }, N}
\end{array}\right] \\
&+\left\{\left[\begin{array}{cc}
\mathbf{I}_{k} & \mathbf{0}_{k \times(m-k)} \\
\mathbf{0}_{(m-k) \times k} & \mathbf{I}_{m-k}
\end{array}\right]-\left[\begin{array}{cc}
\llbracket \boldsymbol{\Gamma}_{F} \rrbracket & \mathbf{0}_{k \times(m-k)} \\
\mathbf{0}_{(m-k) \times k} & \llbracket \mathbf{I}_{m-k} \rrbracket
\end{array}\right]\right\} \\
& \times\left[\begin{array}{c}
\overline{\mathbf{u}}_{F} \\
\mathbf{0}_{(m-k) \times 1}
\end{array}\right] \\
& \underset{\text { Let }}{\Longrightarrow} \mathbf{u}=\llbracket \boldsymbol{\Gamma} \rrbracket \boldsymbol{\Gamma} \mathbf{u}_{\text {normal }}+\left(\mathbf{I}_{m}-\llbracket \boldsymbol{\Gamma} \rrbracket\right) \overline{\mathbf{u}},
\end{aligned}
$$

where $\mathbf{u}_{\text {normal }}=\left[\begin{array}{l}\mathbf{u}_{\text {normal }, F}^{T}, \mathbf{u}_{\text {normal }, N}^{T}\end{array}\right]^{T}, \overline{\mathbf{u}}=\left[\begin{array}{ll}\overline{\mathbf{u}}_{F}^{T} & \mathbf{0}^{T}\end{array}\right]^{T}$,

$$
\begin{aligned}
& \mathbf{u}_{\text {normal }}=\left[\begin{array}{c}
\mathbf{u}_{\text {normal }, F} \\
\mathbf{u}_{\text {normal }, N}
\end{array}\right], \quad \overline{\mathbf{u}}=\left[\begin{array}{c}
\overline{\mathbf{u}}_{F} \\
\mathbf{0}_{(m-k) \times 1}
\end{array}\right], \\
& \boldsymbol{\Gamma}=\left[\begin{array}{cc}
\boldsymbol{\Gamma}_{F} & \mathbf{0}_{k \times(m-k)} \\
\mathbf{0}_{(m-k) \times k} & \mathbf{I}_{m-k}
\end{array}\right], \\
& \llbracket \boldsymbol{\Gamma} \rrbracket=\left[\begin{array}{cc}
\llbracket \boldsymbol{\Gamma}_{F} \rrbracket & \llbracket \mathbf{0}_{k \times(m-k)} \rrbracket \\
\llbracket \mathbf{0}_{(m-k) \times k} \rrbracket & \llbracket \mathbf{I}_{m-k} \rrbracket
\end{array}\right] \\
& =\left[\begin{array}{cc}
\llbracket \boldsymbol{\Gamma}_{F} \rrbracket & \mathbf{0}_{k \times(m-k)} \\
\mathbf{0}_{(m-k) \times k} & \mathbf{I}_{m-k}
\end{array}\right] .
\end{aligned}
$$

In (5), if all actuators are operated normally, then it satisfies the fact that $\boldsymbol{\Gamma}_{F}=\mathbf{I}_{k}$ and $\llbracket \boldsymbol{\Gamma}_{F} \rrbracket=\mathbf{I}_{k}$, and in (6), $\boldsymbol{\Gamma}=\mathbf{I}_{m}$ and $\llbracket \boldsymbol{\Gamma} \rrbracket=\mathbf{I}_{m}$. So $\mathbf{u}=\mathbf{u}_{\text {normal }}$.

From (5), failure-information data for generalizing the faulty dynamic model can be represented as the quantitative value $\gamma_{i}$. Each smart actuator diagnoses its health condition by estimating the value $\gamma_{i}$. By transferring this data, faulttolerant controller can accommodate failures occurring on multiple actuators.

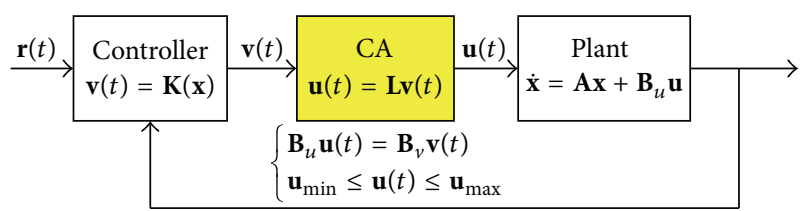

FIGURE 3: The structure of control allocation.

2.2. General Dynamics of Faulty System. Let us consider the following dynamical system:

$$
\dot{\mathbf{x}}=\mathbf{A x}+\mathbf{B}_{u} \mathbf{u}+\mathbf{d}
$$

where $\mathbf{x} \in \mathbf{R}^{n}$ and $\mathbf{u} \in \mathbf{R}^{m}$ are the state and input vectors, respectively. And $\mathbf{B}_{u}$ is the input distribution matrix denoted by $\mathbf{B}_{u}=\left[\mathbf{b}_{u, 1}, \mathbf{b}_{u, 2}, \ldots, \mathbf{b}_{u, m}\right]$. And $\mathbf{d} \in \mathbf{R}^{n}$ is the model uncertainty vector of the system.

By (5), if failures occurred on $k(1 \leq k \leq m)$ actuators, then the system model can be generalized as follows:

$$
\begin{aligned}
\dot{\mathbf{x}}= & \mathbf{A} \mathbf{x}+\mathbf{B}_{u} \mathbf{u}=\mathbf{A} \mathbf{x}+\mathbf{B}_{u}\left[\llbracket \boldsymbol{\Gamma} \rrbracket \mathbf{\Gamma}_{\mathrm{normal}}+\left(\mathbf{I}_{m}-\llbracket \boldsymbol{\Gamma} \rrbracket\right) \overline{\mathbf{u}}\right]+\mathbf{d} \\
= & \mathbf{A x}+\left[\begin{array}{ll}
\mathbf{B}_{F} & \mathbf{B}_{N}
\end{array}\right] \\
& \times\left\{\left[\begin{array}{cc}
\llbracket \boldsymbol{\Gamma}_{F} \rrbracket \boldsymbol{\Gamma}_{F} & \mathbf{0}_{k \times(m-k)} \\
\mathbf{0}_{(m-k) \times k} & \mathbf{I}_{m-k}
\end{array}\right]\left[\begin{array}{c}
\mathbf{u}_{\text {normal }, F} \\
\mathbf{u}_{\text {normal }, N}
\end{array}\right]\right. \\
& \left.+\left[\begin{array}{cc}
\left(\mathbf{I}_{k}-\llbracket \boldsymbol{\Gamma}_{F} \rrbracket\right) & \mathbf{0}_{k \times(m-k)} \\
\mathbf{0}_{(m-k) \times k} & \mathbf{0}_{(m-k) \times(m-k)}
\end{array}\right]\left[\begin{array}{c}
\overline{\mathbf{u}}_{F} \\
\mathbf{0}_{(m-k) \times 1}
\end{array}\right]\right\}+\mathbf{d} \\
= & \mathbf{A x}+\mathbf{B}_{N} \mathbf{u}_{\text {normal }, N} \\
& +\mathbf{B}_{F}\left[\llbracket \boldsymbol{\Gamma}_{F} \rrbracket \boldsymbol{\Gamma}_{F} \mathbf{u}_{\text {normal }, F}+\left(\mathbf{I}_{k}-\llbracket \boldsymbol{\Gamma}_{F} \rrbracket\right) \overline{\mathbf{u}}_{F}\right]+\mathbf{d},
\end{aligned}
$$

where $\mathbf{B}_{F} \in \mathbf{R}^{n \times k}$ and $\mathbf{B}_{N} \in \mathbf{R}^{n \times(m-k)}$ are failure and normal input distribution matrices, respectively; that is, if failures occurred on both $i$ th and $j$ th $(i \leq j)$ actuators, then $\mathbf{B}_{F}=\left[\mathbf{b}_{u, i}, \mathbf{b}_{u, j}\right]$ and $\mathbf{B}_{N}=\left[\mathbf{b}_{u, 1}, \mathbf{b}_{u, 2}, \ldots, \mathbf{b}_{u, i-1}, \mathbf{b}_{u, i+1}\right.$, $\left.\ldots, \mathbf{b}_{u, j-1}, \mathbf{b}_{u, j+1}, \ldots, \mathbf{b}_{u, m}\right]$.

\section{The Proposed Fault-Tolerant Control Allocation Method}

Like modern aircrafts such as the F-18 HARV (High Alpha Research Vehicle) considered in this paper, additional actuators, also called control surfaces, are adopted to achieve high performance by producing additional forces/moments. Redundant actuators also provide opportunities to tolerate failures in actuators by providing additional moments enough to compensate unexpected disturbances generated by faulty actuators. In this section, an additional actuator management method for tolerating actuator failures is proposed by extending a conventional actuator management technique called control allocation (CA).

3.1. Fault-Tolerant Control Allocation Problem. CA is an actuator management technique in overactuated systems. Figure 3 describes the general structure of CA. In this figure, for a virtual-input signal $\mathbf{v}(t) \in \mathbf{R}^{q}$ generated by the 
controller, the control allocator generates the corresponding real control input signal $\mathbf{u}(t) \in \mathbf{R}^{m}$. For the dynamical system represented in (7) with $u_{i, \min } \leq u_{i} \leq u_{i \text {, max }}$, suppose that the equivalent system controlled by the virtual input is represented as follows:

$$
\dot{\mathbf{x}}=\mathbf{A x}+\mathbf{B}_{v} \mathbf{v}
$$

where $\mathbf{B}_{v} \in \mathbf{R}^{n \times q}$ with $q \leq n$ is a full column rank matrix. Then the control allocation problem (CAP) can be stated as follows $[9,10]$.

3.1.1. Control Allocation Problem (CAP). Given a virtual input $\mathbf{v}(t)$, find the optimal actuator commands $\mathbf{u}(t)$ such that the following equation is satisfied:

$$
\begin{aligned}
& \mathbf{B}_{u} \mathbf{u}(t)=\mathbf{B}_{v} \mathbf{v}(t) \\
& \text { subject to } u_{i, \min } \leq u_{i}(t) \leq u_{i, \max } .
\end{aligned}
$$

From (10), the redundant actuator redistribution problem is converted to the problem of finding the optimal inputs.

Similar to the CAP, redistribution of redundant actuators for tolerating actuator failures can also be represented as the optimal reconfiguration input problem. For the generalized faulty system (8) and the corresponding virtual-input system (9), the fault-tolerant control allocation problem (FTCAP) can be represented as follows.

\subsubsection{Fault-Tolerant Control Allocation Problem (FTCAP).} Given a virtual input $\mathbf{v}(t)$, find the optimal reconfigurable actuator commands $\mathbf{u}_{r}(t)$ such that the following equation is satisfied:

$$
\begin{aligned}
& \mathbf{B}_{u}\left[\llbracket \boldsymbol{\Gamma} \rrbracket \boldsymbol{\Gamma} \mathbf{u}_{r}(t)+\left(\mathbf{I}_{m}-\llbracket \Gamma \rrbracket\right) \overline{\mathbf{u}}(t)\right]=\mathbf{B}_{v} \mathbf{v}(t) \\
& \text { subject to } \mathbf{u}_{\min } \leq \mathbf{u}_{r}(t) \leq \mathbf{u}_{\max },
\end{aligned}
$$

where $\mathbf{u}_{\min }$ and $\mathbf{u}_{\max }$ are vectors whose entries are the minimum and the maximum values of each entry of $\mathbf{u}_{r}(t)$, respectively. The methodology of finding $\mathbf{u}_{r}(t)$ that satisfies (11) is proposed in the following subsection.

3.2. The Proposed Reconfiguration Law. From the FTCAP, the reconfiguration law finds the suitable control inputs corresponding to the virtual inputs generated by the controller. Hence, the reconfiguration law can be designed by adding two inputs: (a) the normal control inputs that satisfy the CAP in (10) and (b) the additional inputs that can tolerate the degraded performance due to failures. The following theorem proposes the method of designing the reconfiguration inputs.

Theorem 1. If a control system has $k$-faulty actuators, then reconfiguration law $\mathbf{u}_{r}(t)$ can be obtained as follows:

$$
\mathbf{u}_{r}=\mathbf{u}+\mathbf{u}_{a d d},
$$

where $\mathbf{u} \in \mathbf{R}^{m}$ is the desired control input generated by the conventional CAP and $\mathbf{u}_{\text {add }} \in \mathbf{R}^{m}$ is the additional input that satisfies the following condition:

$$
\begin{aligned}
& \mathbf{B}_{F} \mathbf{u}_{\text {normal }, F}-\mathbf{B}_{F} \llbracket \boldsymbol{\Gamma}_{F} \rrbracket \boldsymbol{\Gamma}_{F} \mathbf{u}_{\text {normal }, F}-\mathbf{B}_{F}\left(\mathbf{I}_{k}-\llbracket \boldsymbol{\Gamma}_{F} \rrbracket\right) \overline{\mathbf{u}}_{F} \\
& \quad-\mathbf{B}_{N} \mathbf{u}_{a d d, N}=\mathbf{0},
\end{aligned}
$$

where

$$
\left.\mathbf{u}_{\text {add }}=\left[\begin{array}{c}
\mathbf{0} \\
\mathbf{u}_{\text {add,N }}
\end{array}\right]\right\}(m-k) \times 1
$$

Proof. Using the proposed reconfiguration law in (12), the FTCAP can be analyzed as follows:

$$
\begin{aligned}
& {\left[\begin{array}{ll}
\mathbf{B}_{F} & \mathbf{B}_{N}
\end{array}\right] \llbracket \boldsymbol{\Gamma} \rrbracket \boldsymbol{\Gamma} \mathbf{u}_{r}+\left[\begin{array}{ll}
\mathbf{B}_{F} & \mathbf{B}_{N}
\end{array}\right]\left(\mathbf{I}_{m}-\llbracket \boldsymbol{\Gamma} \rrbracket\right) \overline{\mathbf{u}}} \\
& =\left[\begin{array}{ll}
\mathbf{B}_{F} & \mathbf{B}_{N}
\end{array}\right] \llbracket \boldsymbol{\Gamma} \rrbracket \boldsymbol{\Gamma}\left(\mathbf{u}+\mathbf{u}_{\mathrm{add}}\right)+\left[\begin{array}{ll}
\mathbf{B}_{F} & \mathbf{B}_{N}
\end{array}\right]\left(\mathbf{I}_{m}-\llbracket \boldsymbol{\Gamma} \rrbracket\right) \overline{\mathbf{u}} \\
& = \\
& \quad\left[\begin{array}{ll}
\mathbf{B}_{F} & \mathbf{B}_{N}
\end{array}\right]\left[\begin{array}{cc}
\llbracket \boldsymbol{\Gamma}_{F} \rrbracket \boldsymbol{\Gamma}_{F} & \mathbf{0}_{k \times(m-k)} \\
\mathbf{0}_{(m-k) \times k} & \mathbf{I}_{m-k}
\end{array}\right]\left\{\left[\begin{array}{l}
\mathbf{u}_{\text {normal }, F} \\
\mathbf{u}_{\text {normal }, N}
\end{array}\right]+\left[\begin{array}{c}
\mathbf{0} \\
\mathbf{u}_{\mathrm{add}, N}
\end{array}\right]\right\} \\
& = \\
& \left.\quad \mathbf{B}_{N} \mathbf{B}_{N}\right]\left[\begin{array}{cc}
\mathbf{I}_{k}-\llbracket \boldsymbol{\Gamma}_{F} \rrbracket & \mathbf{0}_{k \times(m-k)} \\
\mathbf{0}_{(m-k) \times k} & \mathbf{0}_{(m-k) \times(m-k)}
\end{array}\right]\left[\begin{array}{c}
\overline{\mathbf{u}}_{F} \\
\mathbf{0}_{(m-k) \times 1}
\end{array}\right] \\
& +\mathbf{B}_{N} \mathbf{u}_{\mathrm{add}, N} .
\end{aligned}
$$

Substituting (13) into (15), the following equation is satisfied:

$$
\begin{aligned}
\mathbf{B}_{N} \mathbf{u}_{\text {normal }, N}+\mathbf{B}_{F}\left[\llbracket \boldsymbol{\Gamma}_{F} \rrbracket \boldsymbol{\Gamma}_{F} \mathbf{u}_{\text {normal }, F}+\left(\mathbf{I}_{k}-\llbracket \boldsymbol{\Gamma}_{F} \rrbracket\right) \overline{\mathbf{u}}_{F}\right] \\
\quad+\mathbf{B}_{N} \mathbf{u}_{\text {add }, N}=\mathbf{B}_{N} \mathbf{u}_{\text {normal }, N}+\mathbf{B}_{F} \mathbf{u}_{\text {normal }, F} \\
=\mathbf{B}_{u} \mathbf{u}=\mathbf{B}_{v} \mathbf{v} .
\end{aligned}
$$

So the degraded performance can be compensated using the proposed fault-tolerant control allocation method. Hence, the control system can maintain the normal operation.

From Theorem 1, the proposed reconfiguration law can efficiently compensate failures on actuators and the system can maintain the performance of normal operation. It is worth noting that pseudoinverse, constraint optimization, and so forth can be used to find the additional input $\mathbf{u}_{\text {add }, N}$ in (13). Moreover, Buffington and Enns proposed the daisy-chain method that overcomes the actuator saturation problem for the conventional CA [17]. Since actuators are physically operated in limited range, some actuators cannot reach the desired position. This phenomenon is called actuator saturation. Errors between the actual and the desired actuator positions lead to the performance degradation of the control system. In this paper, the results of the daisy-chain method are modified to compensate failures in the following remark.

Remark 2 (fault-tolerant daisy-chain method). Let us separate actuators into $Q$ groups $(Q \leq m)$ as $\mathbf{u}=\left[\mathbf{u}_{1}^{T}, \mathbf{u}_{2}^{T}, \ldots, \mathbf{u}_{Q}^{T}\right]^{T}$. And let us reorder and separate the column vectors of $\mathbf{B}_{u}$ in (8) as $\mathbf{B}_{u}=\left[\mathbf{B}_{1}, \mathbf{B}_{2}, \ldots, \mathbf{B}_{Q}\right]$ along with the corresponding $\mathbf{u}_{l}(l=1,2, \ldots, Q)$. To simplify the problem, all faulty actuators are assumed in Group 1; that is, $\mathbf{u}_{\text {normal, } F} \subset \mathbf{u}_{1}$. Then 
the generalized faulty model represented in (8) can be represented as follows:

$$
\begin{aligned}
& \dot{\mathbf{x}}=\mathbf{A} \mathbf{x}+\mathbf{B}_{u} \mathbf{u}=\mathbf{A} \mathbf{x}+\mathbf{B}_{u}\left[\llbracket \Gamma \rrbracket \Gamma \mathbf{u}_{\text {normal }}+\left(\mathbf{I}_{m}-\llbracket \Gamma \rrbracket\right) \overline{\mathbf{u}}\right]+\mathbf{d} \\
& =\mathbf{A} \mathbf{x}+\left[\begin{array}{cccc}
\mathbf{B}_{1} \\
=\left[\begin{array}{llll}
\mathbf{B}_{F} & \mathbf{B}_{N}
\end{array}\right] & \mathbf{B}_{2} & \cdots & \mathbf{B}_{\mathrm{Q}}
\end{array}\right]
\end{aligned}
$$

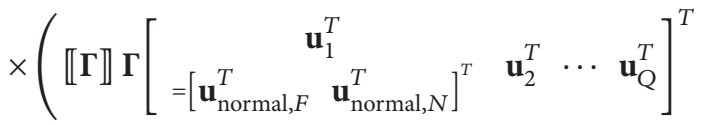

$$
\begin{aligned}
& \left.+\left(\mathbf{I}_{m}-\llbracket \boldsymbol{\Gamma} \rrbracket\right) \overline{\mathbf{u}}\right)+\mathbf{d} \\
& =\mathbf{A x}+\sum_{i=2}^{Q} \mathbf{B}_{i} \mathbf{u}_{i} \\
& +\left[\begin{array}{ll}
\mathbf{B}_{F} & \mathbf{B}_{N}
\end{array}\right]\left\{\left[\begin{array}{cc}
\llbracket \boldsymbol{\Gamma}_{F} \rrbracket \boldsymbol{\Gamma}_{F} & \mathbf{0}_{k \times\left(m_{1}-k\right)} \\
\mathbf{0}_{\left(m_{1}-k\right) \times k} & \mathbf{I}_{m_{1}-k}
\end{array}\right]\left[\begin{array}{l}
\mathbf{u}_{\text {normal }, F} \\
\mathbf{u}_{\text {normal }, N}
\end{array}\right]\right. \\
& +\left[\begin{array}{cc}
\left(\mathbf{I}_{k}-\llbracket \boldsymbol{\Gamma}_{F} \rrbracket\right) & \mathbf{0}_{k \times\left(m_{1}-k\right)} \\
\mathbf{0}_{\left(m_{1}-k\right) \times k} & \mathbf{0}_{\left(m_{1}-k\right) \times\left(m_{1}-k\right)}
\end{array}\right] \\
& \left.\times\left[\begin{array}{c}
\overline{\mathbf{u}}_{F} \\
\mathbf{0}_{\left(m_{1}-k\right) \times 1}
\end{array}\right]\right\}+\mathbf{d} \\
& =\mathbf{A} \mathbf{x}+\sum_{i=2}^{Q} \mathbf{B}_{i} \mathbf{u}_{i}+\mathbf{B}_{N} \mathbf{u}_{\text {normal }, N} \\
& +\mathbf{B}_{F}\left[\llbracket \boldsymbol{\Gamma}_{F} \rrbracket \boldsymbol{\Gamma}_{F} \mathbf{u}_{\text {normal, } F}+\left(\mathbf{I}_{k}-\llbracket \boldsymbol{\Gamma}_{F} \rrbracket\right) \overline{\mathbf{u}}_{F}\right]+\mathbf{d},
\end{aligned}
$$

where $m_{1}$ is the number of actuators in Group 1.

Theorem 3. For the generalized faulty system (17), the reconfiguration law $\mathbf{u}_{r}(t)$ can be obtained as follows:

$$
\mathbf{u}_{r}\left(=\left[\begin{array}{llll}
\mathbf{u}_{r, 1}^{T} & \mathbf{u}_{r, 2}^{T} & \cdots & \mathbf{u}_{r, \mathrm{Q}}^{T}
\end{array}\right]^{T}\right)=\mathbf{u}+\mathbf{u}_{a d d}
$$

where $\mathbf{u}_{a d d}=\left[\mathbf{u}_{a d d, 1}^{T}, \mathbf{u}_{a d d, 2}^{T}, \ldots, \mathbf{u}_{a d d, Q}^{T}\right]^{T}$ is the additional inputs provided by redistributing the redundant actuators to compensate for failures and satisfies the following conditions.

(a) Let $\mathbf{u}_{a d d, 1}=\left[\mathbf{0}^{T}, \mathbf{u}_{a d d, N}^{T}\right]^{T}$, and let $\mathbf{P}_{N}$ be the right inverse of $\mathbf{B}_{N}$. Then

$$
\begin{gathered}
\mathbf{u}_{a d d, N}=\operatorname{sat}_{\mathbf{u}_{a d d, N}}\left(\mathbf { P } _ { N } \left(\mathbf{B}_{F} \mathbf{u}_{\text {normal }, F}-\mathbf{B}_{F} \llbracket \boldsymbol{\Gamma}_{F} \rrbracket \boldsymbol{\Gamma}_{F} \mathbf{u}_{\text {normal }, F}\right.\right. \\
\left.\left.-\mathbf{B}_{F}\left(\mathbf{I}_{k}-\llbracket \boldsymbol{\Gamma}_{F} \rrbracket\right) \overline{\mathbf{u}}_{F}\right)\right) .
\end{gathered}
$$

(b) For $\mathbf{P}_{l}(l=2,3, \ldots, Q)$, the right inverse of $\mathbf{B}_{l}$,

$$
\begin{gathered}
\mathbf{u}_{a d d, l}=\operatorname{sat}_{\mathbf{u}_{a d d, l}}\left(\mathbf { P } _ { l } \left(\mathbf{B}_{F} \mathbf{u}_{\text {normal }, F}-\mathbf{B}_{F} \llbracket \boldsymbol{\Gamma}_{F} \rrbracket \boldsymbol{\Gamma}_{F} \mathbf{u}_{\text {normal }, F}\right.\right. \\
-\mathbf{B}_{F}\left(\mathbf{I}_{k}-\llbracket \boldsymbol{\Gamma}_{F} \rrbracket\right) \overline{\mathbf{u}}_{F}-\mathbf{B}_{N} \mathbf{u}_{a d d, N} \\
\left.\left.-\sum_{i=2}^{l-1} \mathbf{B}_{i} \mathbf{u}_{r, i}\right)\right) .
\end{gathered}
$$

(It is worth noting that $\mathbf{u}_{a d d, N}$ is used to compensate the effect of failure actuators. If $\mathbf{u}_{a d d, N}$ cannot compensate perfectly due to saturation, then $\mathbf{u}_{\text {add,2 }}$ tries to compensate the remainder. Similarly, $\mathbf{u}_{a d d, l}(l=3,4, \ldots, Q)$ is used, if $\mathbf{u}_{a d d, l-1}$ is saturated at the upper or the lower limited position.)

Proof. If the system has $k$-faulty actuators, then the system dynamics can be generalized as (17). Using the reconfiguration law proposed in (18), the faulty actuator term of (17) satisfies the following:

$$
\begin{aligned}
& \sum_{i=2}^{Q} \mathbf{B}_{i} \mathbf{u}_{r, i}+\mathbf{B}_{1}\left[\llbracket \Gamma \rrbracket \Gamma \mathbf{u}_{r, 1}+\left(\mathbf{I}_{m}-\llbracket \Gamma \rrbracket\right) \overline{\mathbf{u}}\right] \\
& =\sum_{i=2}^{Q} \mathbf{B}_{i} \mathbf{u}_{r, i}+\left[\begin{array}{ll}
\mathbf{B}_{F} & \mathbf{B}_{N}
\end{array}\right]\left[\begin{array}{cc}
\llbracket \boldsymbol{\Gamma}_{F} \rrbracket \boldsymbol{\Gamma}_{F} & \mathbf{0}_{k \times\left(m_{1}-k\right)} \\
\mathbf{0}_{\left(m_{1}-k\right) \times k} & \mathbf{I}_{m_{1}-k}
\end{array}\right] \\
& \times\left\{\left[\begin{array}{c}
\mathbf{u}_{\text {normal }, F} \\
\mathbf{u}_{\text {normal }, N}
\end{array}\right]+\left[\begin{array}{c}
\mathbf{0}_{k \times 1} \\
\mathbf{u}_{\text {add }, N}
\end{array}\right]\right\}+\left[\begin{array}{ll}
\mathbf{B}_{F} & \mathbf{B}_{N}
\end{array}\right] \\
& \times\left[\begin{array}{cc}
\left(\mathbf{I}_{k}-\llbracket \boldsymbol{\Gamma}_{F} \rrbracket\right) & \mathbf{0}_{k \times\left(m_{1}-k\right)} \\
\mathbf{0}_{\left(m_{1}-k\right) \times k} & \mathbf{0}_{\left(m_{1}-k\right) \times\left(m_{1}-k\right)}
\end{array}\right]\left[\begin{array}{c}
\overline{\mathbf{u}}_{F} \\
\mathbf{0}_{\left(m_{1}-k\right) \times 1}
\end{array}\right] \\
& =\sum_{i=2}^{Q} \mathbf{B}_{i} \mathbf{u}_{i}+\sum_{i=2}^{Q} \mathbf{B}_{i} \mathbf{u}_{\mathrm{add}, i} \\
& +\mathbf{B}_{F}\left[\llbracket \boldsymbol{\Gamma}_{F} \rrbracket \boldsymbol{\Gamma}_{F} \mathbf{u}_{\text {normal }, F}+\left(\mathbf{I}_{k}-\llbracket \boldsymbol{\Gamma}_{F} \rrbracket\right) \overline{\mathbf{u}}_{F}\right] \\
& +\mathbf{B}_{N}\left(\mathbf{u}_{\text {normal }, N}+\mathbf{u}_{\mathrm{add}, N}\right) .
\end{aligned}
$$

If the conditions (a) and (b) are satisfied, then the additional inputs try to reduce the errors due to saturated actuators as well as faulty actuators:

$$
\begin{aligned}
\mathbf{B}_{N} \mathbf{u}_{\mathrm{add}, N}+\sum_{i=2}^{Q} \mathbf{B}_{i} \mathbf{u}_{\mathrm{add}, i} \approx & \mathbf{B}_{F} \mathbf{u}_{\mathrm{normal}, F} \\
& -\mathbf{B}_{F} \llbracket \boldsymbol{\Gamma}_{F} \rrbracket \boldsymbol{\Gamma}_{F} \mathbf{u}_{\mathrm{normal}, F} \\
& -\mathbf{B}_{F}\left(\mathbf{I}_{k}-\llbracket \boldsymbol{\Gamma}_{F} \rrbracket\right) \overline{\mathbf{u}}_{F} .
\end{aligned}
$$


If it is assumed that (22) is satisfied, that is, errors are compensated by redistributing the redundant actuators, then (21) can be represented as follows:

$$
\begin{aligned}
\sum_{i=2}^{Q} \mathbf{B}_{i} \mathbf{u}_{i}+\sum_{i=2}^{Q} \mathbf{B}_{i} \mathbf{u}_{\mathrm{add}, i}+\mathbf{B}_{F}\left[\llbracket \boldsymbol{\Gamma}_{F} \rrbracket \boldsymbol{\Gamma}_{F} \mathbf{u}_{\text {normal }, F}+\left(\mathbf{I}_{k}-\llbracket \boldsymbol{\Gamma}_{F} \rrbracket\right) \overline{\mathbf{u}}_{F}\right] \\
\quad+\mathbf{B}_{N}\left(\mathbf{u}_{\text {normal }, N}+\mathbf{u}_{\mathrm{add}, N}\right) \\
=\mathbf{B}_{N} \mathbf{u}_{\text {normal }, N}+\mathbf{B}_{F} \mathbf{u}_{\text {normal }, F}+\sum_{i=2}^{Q} \mathbf{B}_{i} \mathbf{u}_{i} \\
=\mathbf{B}_{1} \mathbf{u}_{1}+\sum_{i=2}^{Q} \mathbf{B}_{i} \mathbf{u}_{i}=\sum_{i=1}^{Q} \mathbf{B}_{i} \mathbf{u}_{i}=\mathbf{B}_{u} \mathbf{u}=\mathbf{B}_{v} \mathbf{v} .
\end{aligned}
$$

So using the fault-tolerant daisy-chain method proposed in (18)-(20), the degraded performance can be compensated. Hence, the control system can maintain the normal operation.

\section{Simulation Results}

4.1. Problem Description. In this section, the performance of the proposed fault-tolerant control allocation technique is evaluated with application to the F-18 HARV aircraft. Like conventional aircrafts, the F-18 HARV adopts ailerons, stabilators, and rudders as primary control surfaces. Indeed, the aircraft also takes six vanes to produce additional moments by vectoring thrust [15]. Hence, the aircraft can overcome stall situation and achieve high angle-of-attack flight ability. The added control surfaces (vanes) also provide an opportunity to improve the fault-tolerance ability that will be evaluated in this section. The structure of the F-18 HARV is described in Figure 4.
TABLE 1: Nomenclatures of control surface [16].

\begin{tabular}{ll}
\hline Symbol & Description \\
\hline$\delta_{\mathrm{SS}}$ & Deflection of symmetric stabilators \\
$\delta_{\mathrm{DS}}$ & Deflection of differential stabilators \\
$\delta_{R}$ & Deflection of symmetric rudders \\
$\delta_{A}$ & Deflection of differential ailerons \\
$\delta_{\text {PTV }}$ & Pitch thrust vectoring nozzle deflection \\
$\delta_{\text {RTV }}$ & Differential pitch thrust vectoring nozzle deflection \\
$\delta_{\text {YTV }}$ & Yaw thrust vectoring nozzle deflection \\
\hline
\end{tabular}

TABLE 2: Control surface model data [16].

\begin{tabular}{lccc}
\hline $\begin{array}{l}\text { Control } \\
\text { surface }\end{array}$ & Transfer function & Rate limits & $\begin{array}{c}\text { Position } \\
\text { limits }\end{array}$ \\
\hline Ailerons & $\frac{75^{2}}{s^{2}+2(0.59)(75)+75^{2}}$ & $\pm 100 \mathrm{deg} / \mathrm{sec}$ & $\pm 27.5 \mathrm{deg}$ \\
Stabilators & $\frac{30.8^{2}}{s^{2}+2(0.51)(30.8)+30.8^{2}}$ & $\pm 40 \mathrm{deg} / \mathrm{sec}$ & $-10.5 \sim 24 \mathrm{deg}$ \\
Rudders & $\frac{72^{2}}{s^{2}+2(0.69)(72)+72^{2}}$ & $\pm 30 \mathrm{deg} / \mathrm{sec}$ & $\pm 30 \mathrm{deg}$ \\
$\begin{array}{l}\text { Pitch } \\
\text { vanes }\end{array}$ & $\frac{75^{2}}{s^{2}+2(0.59)(75)+75^{2}}$ & $\pm 80 \mathrm{deg} / \mathrm{sec}$ & $-25 \sim 20 \mathrm{deg}$ \\
Yaw vanes & $\frac{75^{2}}{s^{2}+2(0.59)(75)+75^{2}}$ & $\pm 80 \mathrm{deg} / \mathrm{sec}$ & $-20 \sim 10 \mathrm{deg}$ \\
\hline
\end{tabular}

In this simulation, two failure scenarios are considered: one with $50 \%$ of LoE failure on the left aileron at $3.5 \mathrm{sec}$ and LiP failure on the left stabilator at $8 \mathrm{sec}$ and the other with float failure on the left aileron at $3.5 \mathrm{sec}$ and hardover failure on the left stabilator at $8 \mathrm{sec}$. In both scenarios, the aircraft performs turn reversal maneuver are achieved by rolling the aircraft to $60 \mathrm{deg}$ to the left and then to $60 \mathrm{deg}$ to the right. Since failures break the symmetry of the aircraft, the longitudinal and lateral/directional dynamics are coupled. The linearized model of the aircraft at Mach Mach 0.6 and altitude $30,000 \mathrm{ft}$ is represented as follows [16]:

$$
\begin{aligned}
{\left[\begin{array}{c}
\dot{\alpha} \\
\dot{q}
\end{array}\right] } & =\left[\begin{array}{cc}
-0.5088 & 0.9940 \\
-1.1310 & -0.2804
\end{array}\right]\left[\begin{array}{c}
\alpha \\
q
\end{array}\right]+\left[\begin{array}{cc}
-0.09277 & -0.01787 \\
-6.573 & -1.525
\end{array}\right]\left[\begin{array}{c}
\delta_{\mathrm{SS}} \\
\delta_{\mathrm{PTV}}
\end{array}\right], \\
{\left[\begin{array}{c}
\dot{\beta} \\
\dot{p} \\
\dot{r}
\end{array}\right]=} & {\left[\begin{array}{cccc}
-0.11180 & 0.09363 & -0.99490 \\
-10.22000 & -1.169000 & 0.43180 \\
2.20100 & -0.009853 & -0.10560
\end{array}\right]\left[\begin{array}{c}
\beta \\
p \\
r
\end{array}\right] } \\
& +\left[\begin{array}{ccccc}
-0.009231 & -0.004885 & 0.01262 & 0.0000 & 0.008328 \\
9.02 & 11.06 & 0.842 & 0.7304 & 0.05522 \\
0.2957 & -0.2637 & -0.6841 & -0.001928 & -0.757
\end{array}\right]\left[\begin{array}{c}
\delta_{\mathrm{DS}} \\
\delta_{A} \\
\delta_{R} \\
\delta_{\mathrm{RTV}} \\
\delta_{\mathrm{YTV}}
\end{array}\right],
\end{aligned}
$$

where $\alpha$ is the angle-of-attack ( $\mathrm{rad})$ and $\beta$ is sideslip ( $\mathrm{rad})$. And $p, q$, and $r$ are roll, pitch, and yaw angular velocities $(\mathrm{rad} / \mathrm{sec})$, respectively. $\delta$ denotes the deflections ( $\mathrm{rad})$ of control surfaces in the F-18 HARV and the subscripts of $\delta$ are represented in Table 1. And Table 2 shows the low-order actuator models and constraints. Moreover, in Figure 4, thrust vectoring nozzle deflections can be obtained by combination of vanes circling the edge of engines. In this simulation, the dynamic inversion controller that satisfies the Level 1 flight requirements is designed to achieve the turn reversal maneuver $[18,19]$. Moreover, it is assumed that maximally $5 \%$ of random disturbances of deflections of control surfaces are injected as uncertainties.

4.2. Simulation Results. The results of the first scenario are shown in Figures 5-7. Due to the injected LoE failure in 


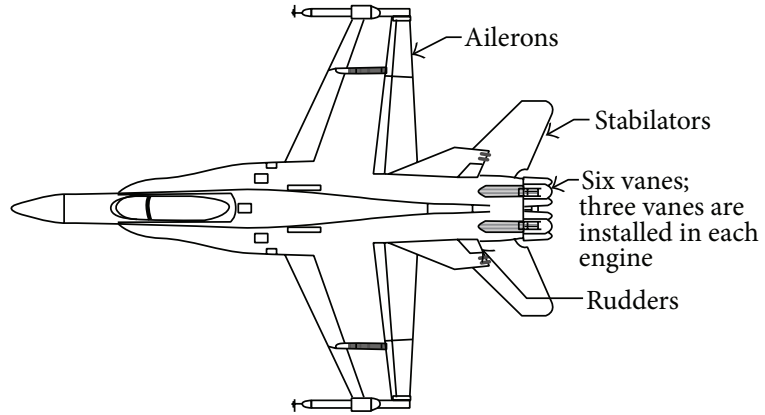

(a)

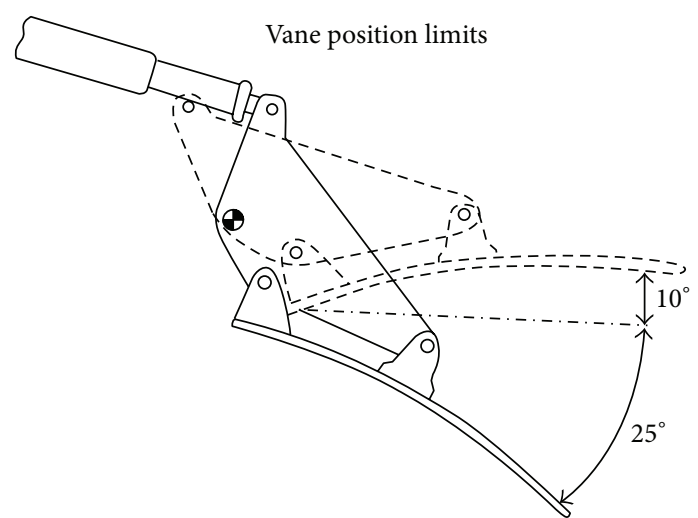

(b)

FIgURE 4: The structure of the F-18 HARV [15].

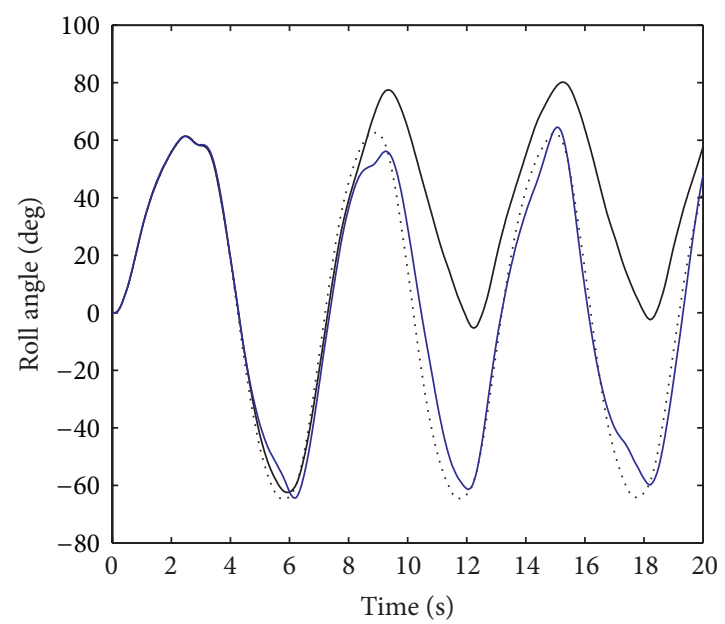

(a) Roll angle

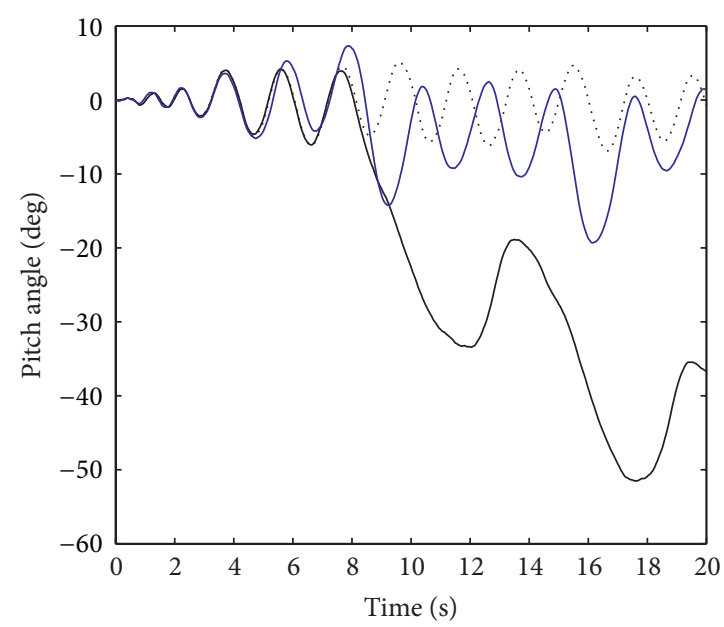

(b) Pitch angle

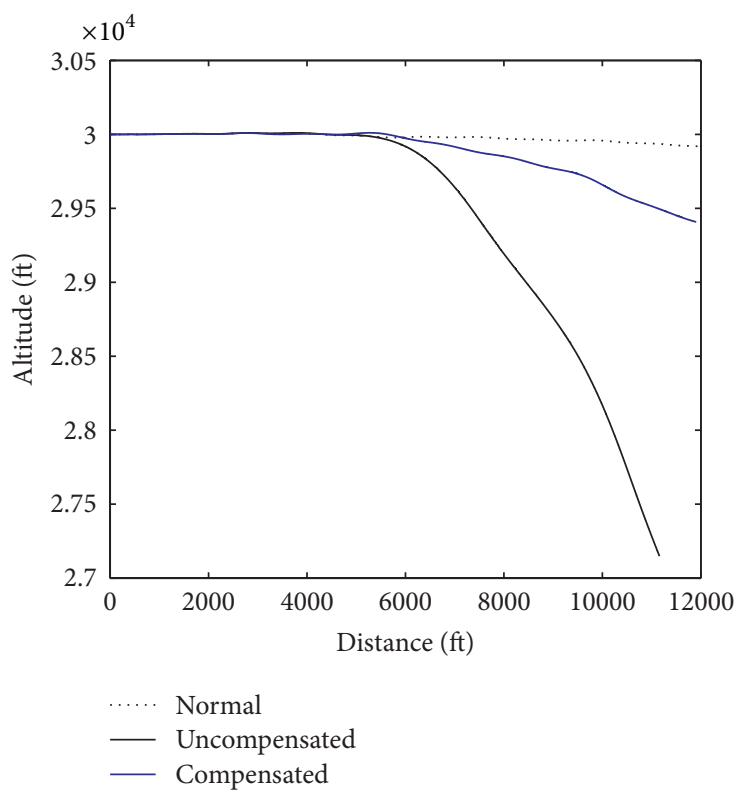

(c) Flight path

FIGURE 5: The results of the turn reversal maneuver with/without reconfiguration. 


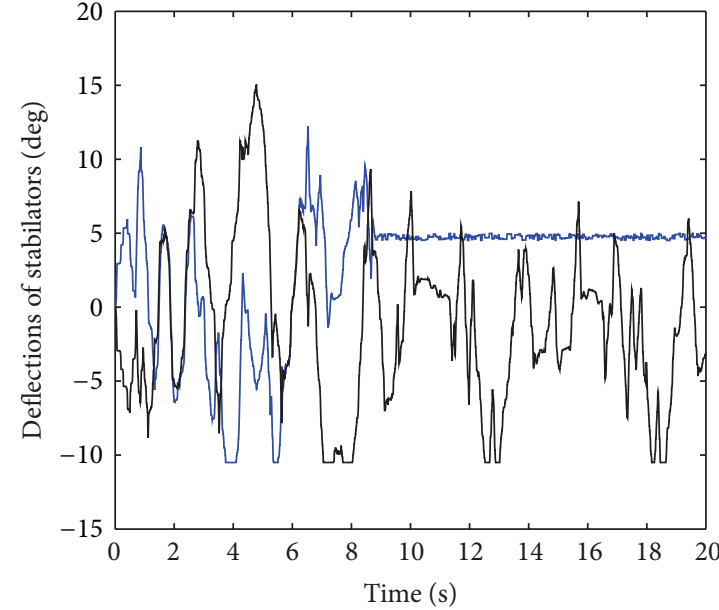

(a)

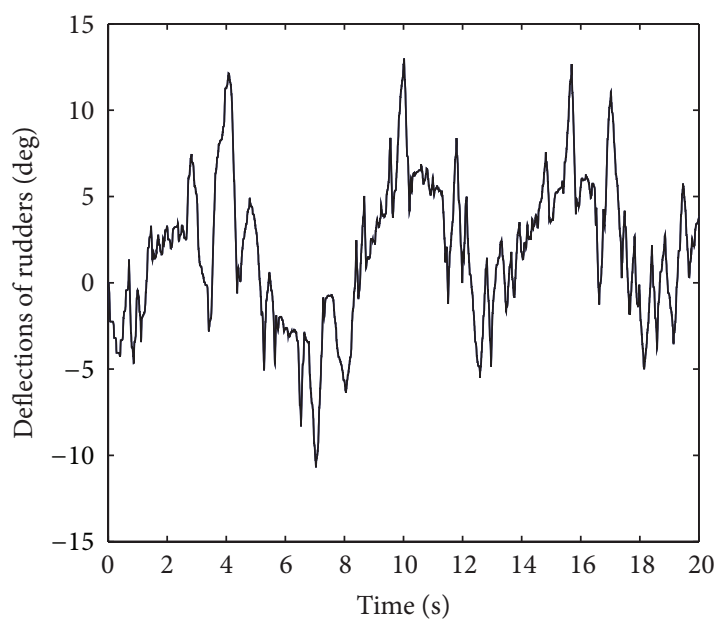

(c)

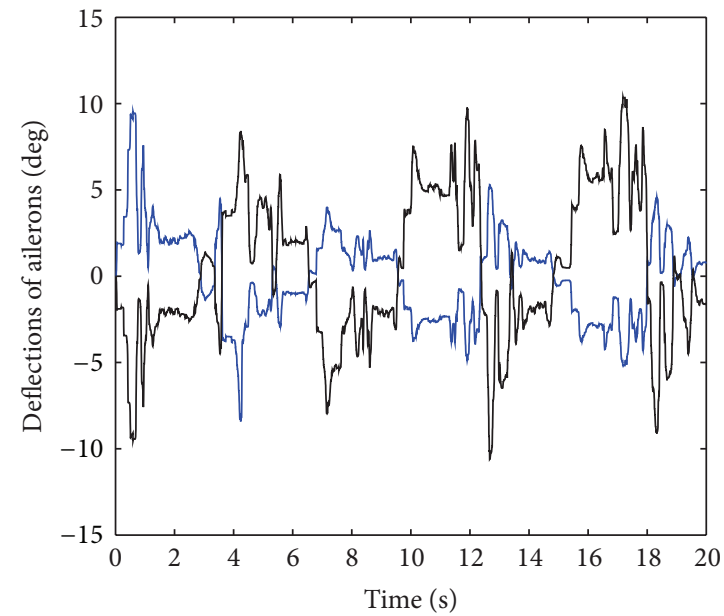

(b)

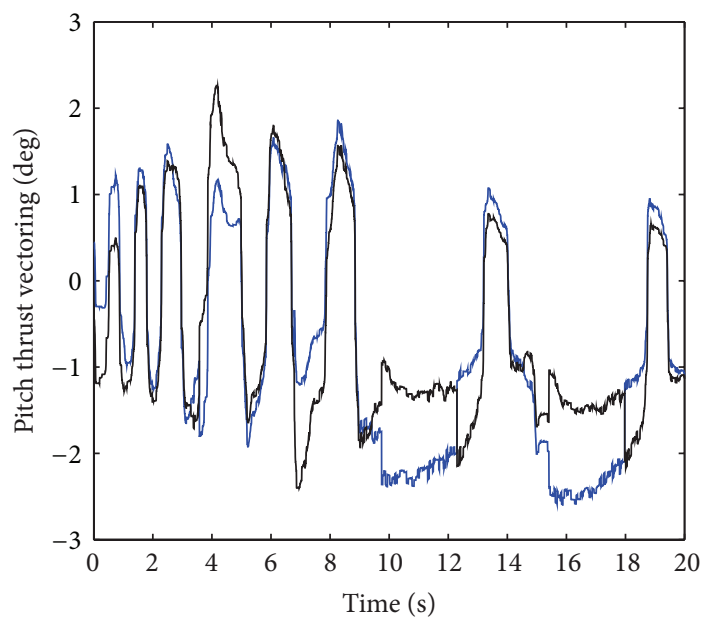

(d)

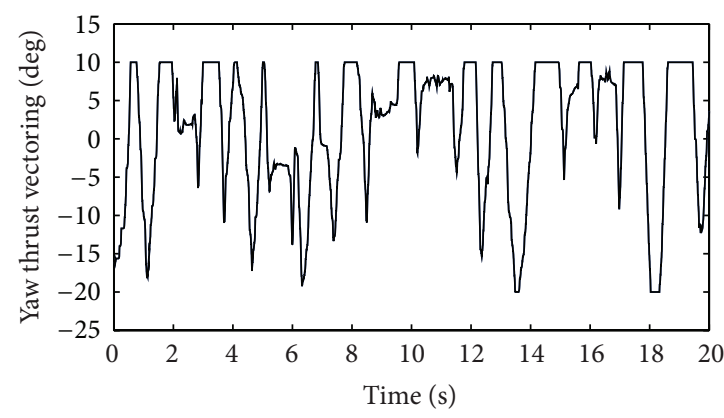

Left
- Right

(e)

FIgURE 6: The deflections of control surfaces without reconfiguration. 


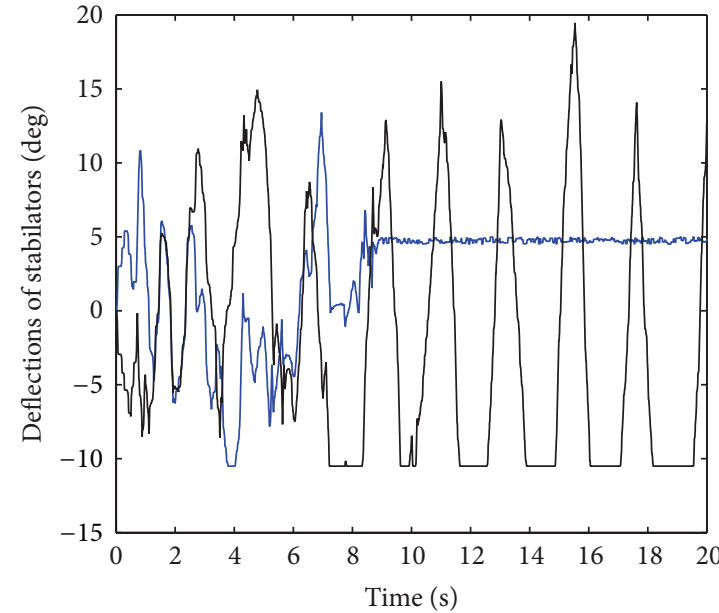

(a)

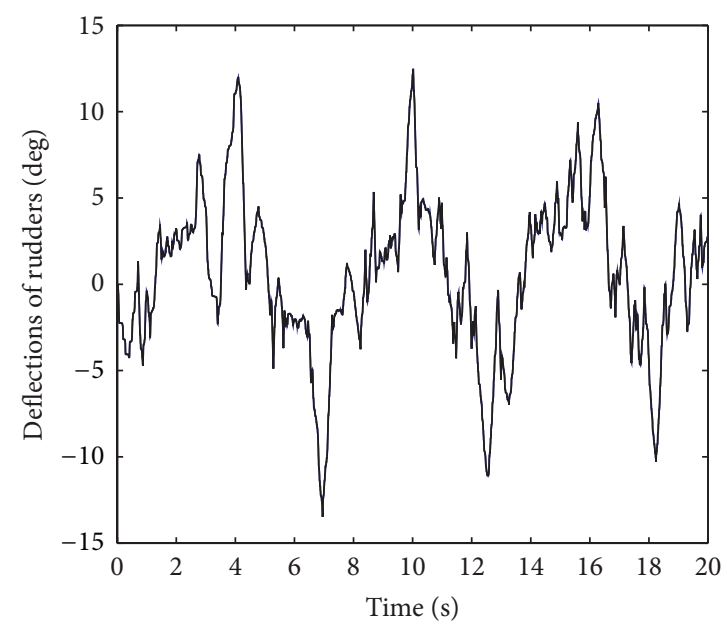

(c)

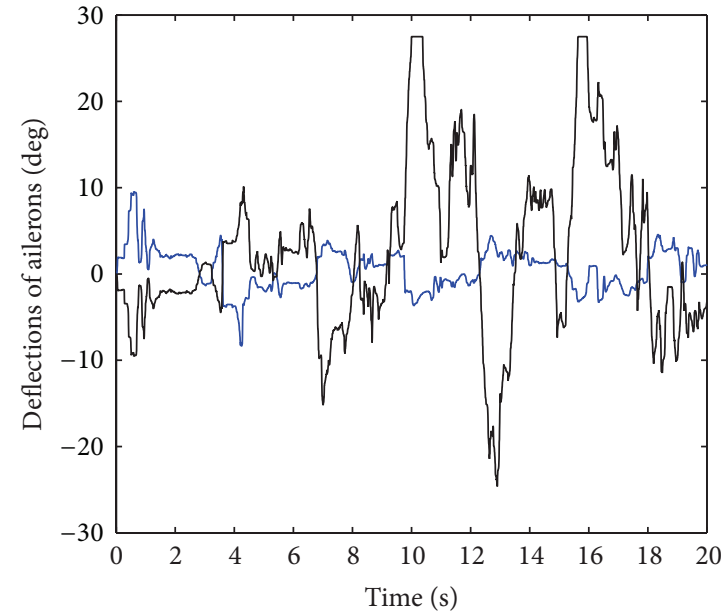

(b)

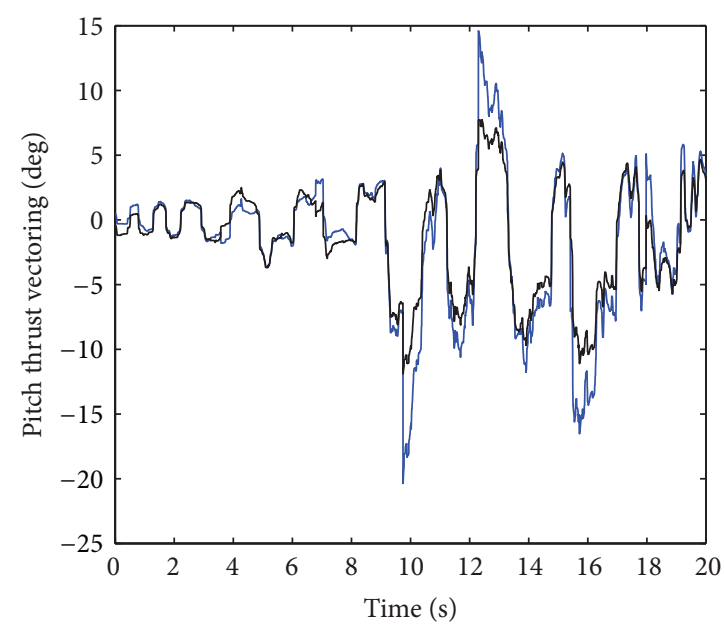

(d)

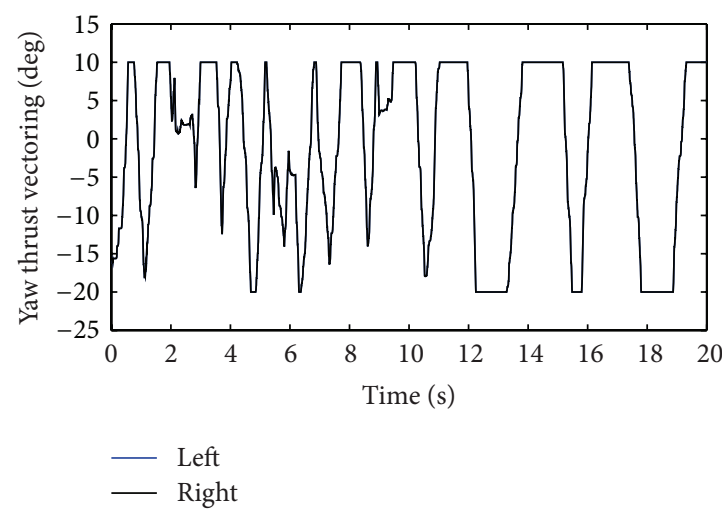

(e)

Figure 7: The deflections of control surfaces with reconfiguration. 


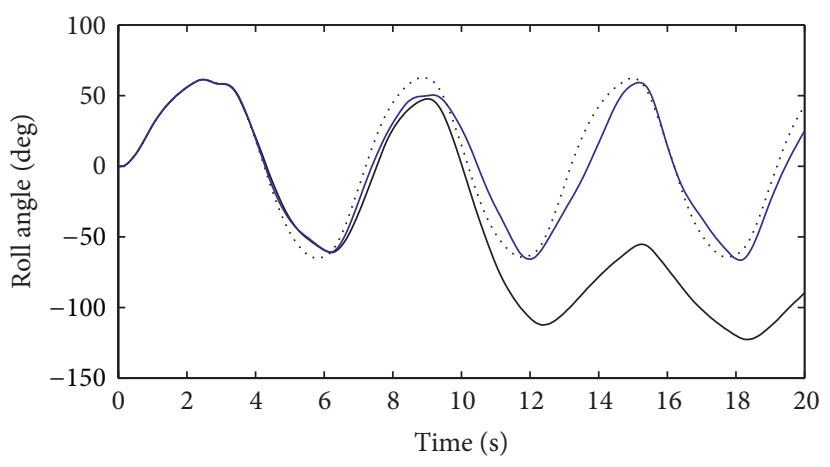

(a) Roll angle

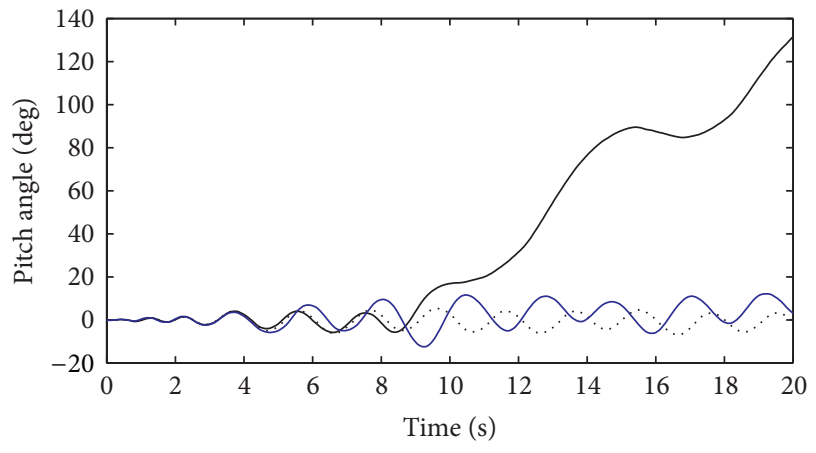

(b) Pitch angle

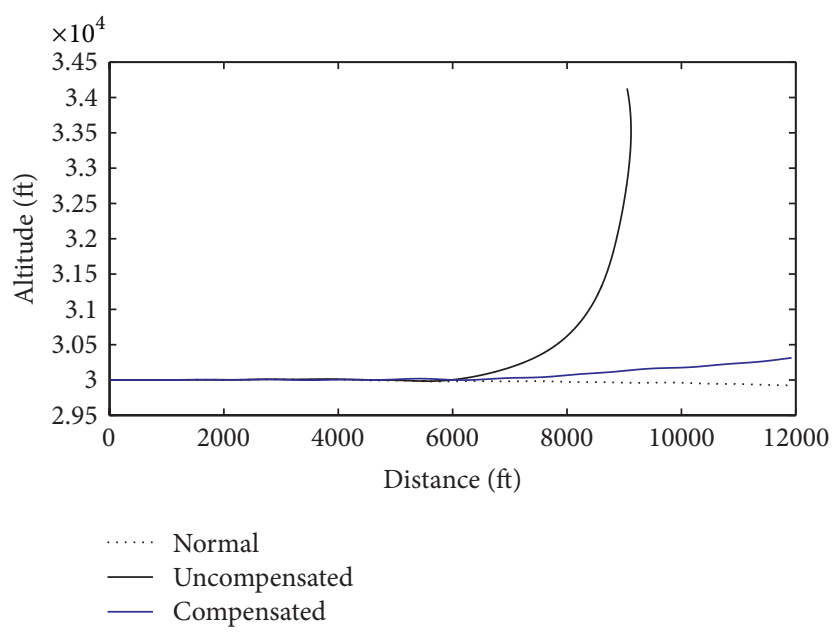

(c) Flight path

FIGURE 8: The results of the turn reversal maneuver with/without reconfiguration.

$3.5 \mathrm{sec}$, the turn reversal maneuver performance is a little degraded. However, locking at one position (near $-3.5 \mathrm{deg}$ ) of the left stabilator due to the LiP failure in $8.0 \mathrm{sec}$ leads to significant degradation of the performance. Since failure on one surface breaks symmetric geometry of the aircraft along with longitudinal axis, it makes the system unstable in longitudinal dynamics (Figure 5(b)). However, using the proposed reconfiguration algorithm, the aircraft can maintain the normal performance. The proposed FTCA method redistributes the redundant actuators in order to compensate the effect of failures on both lateral and longitudinal axes. Figures 6-7 show the deflections of control surfaces in faulty and reconfiguration cases, respectively. In Figure 6, the left aileron is deflected with $50 \%$ of degraded performance in $3.5 \mathrm{sec}$ and the left stabilator locks around $10.5 \mathrm{deg}$ in $8 \mathrm{sec}$. To achieve lateral and longitudinal stability of the faulty aircraft, the deflections of the right stabilator, aileron, and pitch thrust vectoring significantly increased as shown in Figure 7.

Figures 8-10 show the results of the second failure scenario. Similar to the first scenario, the performance of the aircraft is degraded due to the injected float failure on the left aileron in $3.5 \mathrm{sec}$ and hardover failure on the left stabilator in $8.0 \mathrm{sec}$. In particular, the aircraft starts to diverge in longitudinal axis around $8.0 \mathrm{sec}$. In contrast to the without reconfiguration case, the aircraft can maintain the normal performance in both lateral and longitudinal axes by applying the proposed FTCA method. The deflections of control surfaces for faulty and reconfiguration cases are shown in Figures 9-10, respectively. In Figure 9, the left aileron locks the equilibrium position $(0 \mathrm{deg})$ in $3.5 \mathrm{sec}$ and the deflection of the left stabilator diverges to the limit position $-10.5 \mathrm{deg}$ in $8 \mathrm{sec}$. Similar to Scenario I, Figure 10 shows that the deflections of the right stabilator, aileron, and pitch thrust vectoring significantly increase to achieve lateral and longitudinal stability of the faulty aircraft.

Hence, by redistributing the redundant control surfaces based on the proposed FTCA, the aircraft can maintain the normal performance without considering the stability of the reconfigured closed-loop system or predesigning all possible failure models. 


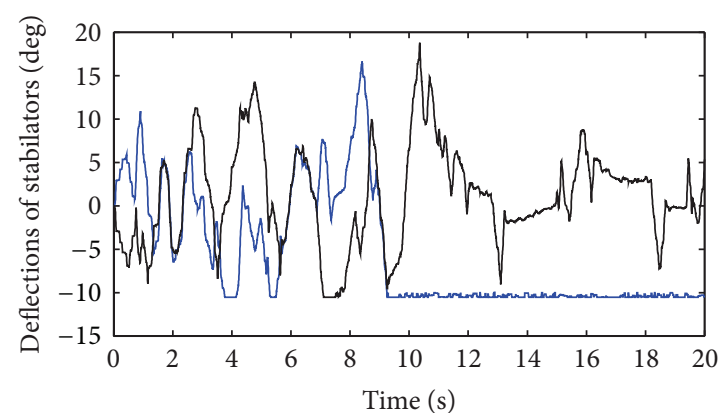

(a)

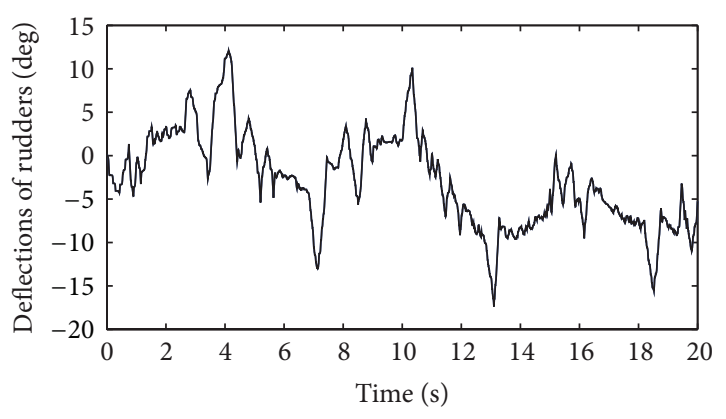

(c)

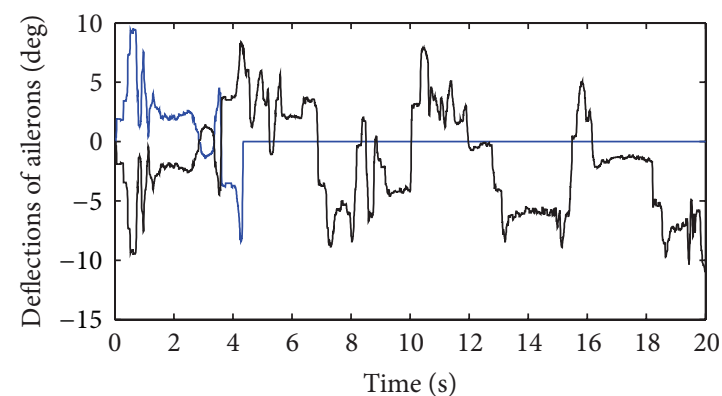

(b)

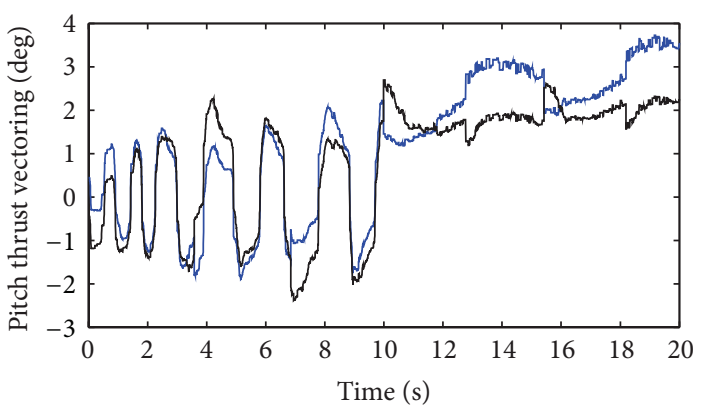

(d)

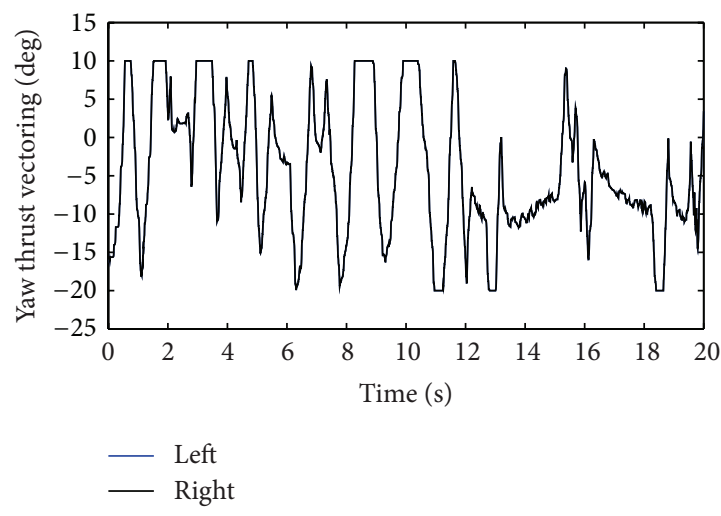

(e)

Figure 9: The deflections of control surfaces without reconfiguration.

\section{Concluding Remarks}

This paper proposed the networked fault-tolerant control method based on control allocation. Using the transmitted condition data diagnosed by the local devices in network environment, the proposed FTC redistributes the redundant actuators in order to tolerate failures on several actuators. To analyze the effects of actuator failure, this paper proposes the general model of the faulty system firstly. From the general failure model, the effect of failure can be represented by a quantitative value $\gamma_{i}$. And then, using this value, the proposed FTCA method achieved by redistributing the redundant actuators can accommodate failures efficiently. The performance of the proposed method has been evaluated with a modern high performance aircraft. A set of simulation results shows that the proposed method can effectively accommodate the actuator failures.

However, the proposed fault-tolerant control allocation is analyzed for a linearized system. Actually, tolerating faults highly depends on the relationship between faulty states and controllable normal inputs. For future work, the proposed method will be extended for nonlinear systems. Moreover, it is planned to implement in real-time nonlinear systems. 


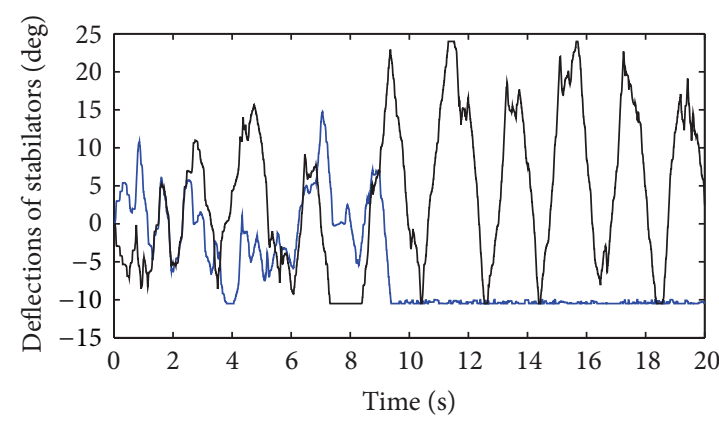

(a)

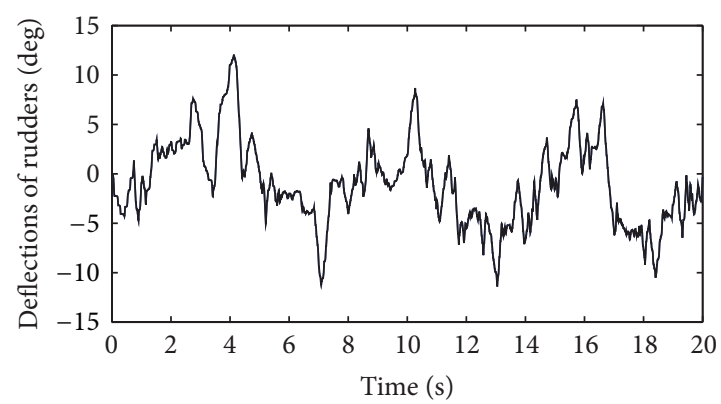

(c)

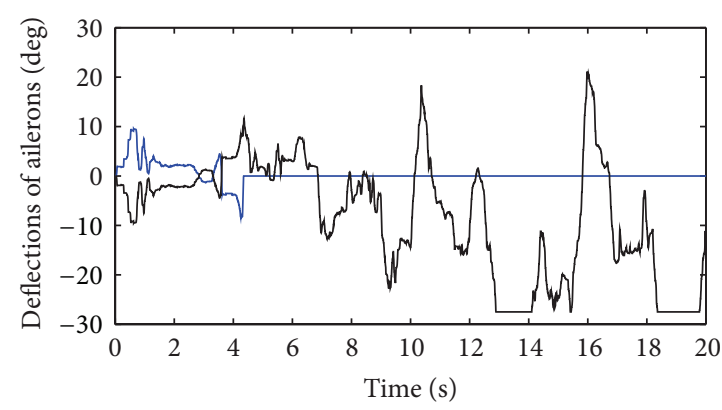

(b)

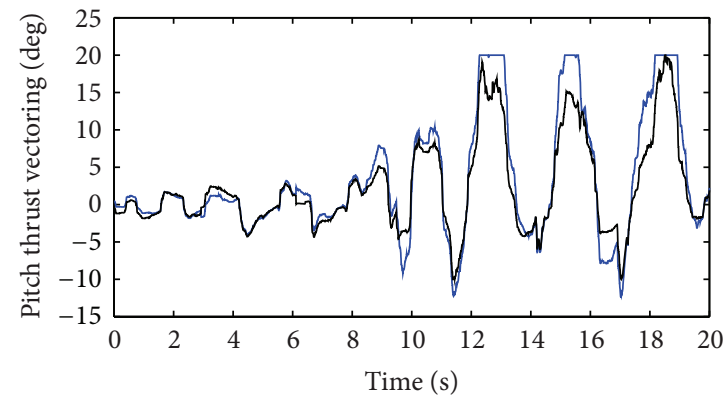

(d)

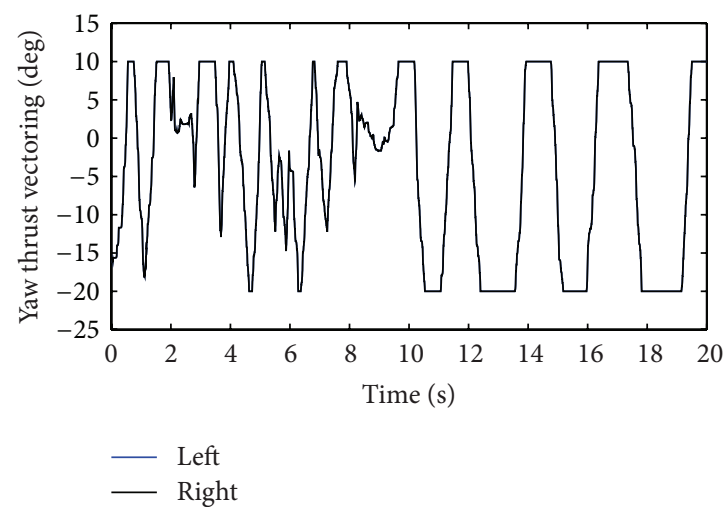

(e)

FIGURE 10: The deflections of control surfaces with reconfiguration.

\section{Conflict of Interests}

The authors declare that there is no conflict of interests regarding the publication of this paper.

\section{Acknowledgment}

This research was supported by the International Research \& Development Program of the National Research Foundation of Korea (NRF) funded by the Ministry of Science, ICT and Future Planning of Korea (2012K1A3A7A03057508).

\section{References}

[1] Z. Gao and P. J. Antsaklis, "Stability of the pseudo-inverse method for reconfigurable control systems," International Journal of Control, vol. 53, no. 3, pp. 717-729, 1991.
[2] K. J. Åström and B. Wittenmark, Adaptive Control, AddisonWesley, Reading, Mass, USA, 2nd edition, 1995.

[3] I. Yang, Y.-J. Kim, and D. Lee, "Actuator failure diagnosis and accommodation using sliding mode control for submersible vehicle," Journal of Institute of Control, Robotics and Systems, vol. 16, no. 7, pp. 661-667, 2010.

[4] J. D. Bošković and R. K. Mehra, "A multiple model-based reconfigurable flight control system design," in Proceedings of the 37th IEEE Conference on Decision \& Control (CDC), pp. 4503-4508, IEEE, Tampa, Fla, USA, December 1998.

[5] D. Enns, "Control allocation approaches," in Proceedings of the AIAA Guidance, Navigation and Control Conference and Exhibit, pp. 98-108, 1998.

[6] J. Buffington, P. Chandler, and M. Pachter, "On-line system identification for aircraft with distributed control effectors," International Journal of Robust and Nonlinear Control, vol. 9, no. 14, pp. 1033-1049, 1999. 
[7] H. Alwi and C. Edwards, "Fault tolerant control using sliding modes with on-line control allocation," Automatica, vol. 44, no. 7, pp. 1859-1866, 2008.

[8] K. Ahmed and T. Didier, "Reconfigurable control design for over-actuated systems based on reliability indicators," in Proceedings of the Conference on Control and Fault Tolerant Systems, Nice, France, October 2010.

[9] I. Yang, D. Kim, and D. Lee, "Fault-tolerant control strategy based on control allocation using smart actuators," in Proceedings of the Conference on Control and Fault-Tolerant Systems, pp. 377-381, Nice, France, October 2010.

[10] I. Yang, D. Kim, and D. Lee, "Fault-Tolerant networked control systems using control allocation for failures in multiple control surfaces," Journal of Institute of Control, Robotics and Systems, vol. 17, no. 11, pp. 1067-1073, 2011.

[11] Y. Zhang and J. Jiang, "Bibliographical review on reconfigurable fault-tolerant control systems," in Proceedings of the 5th IFAC Symposium on Fault Detection, Supervision and Safety for Technical Processes, pp. 265-276, Washington, DC, USA, 2003.

[12] D. Lee, Distributed real-time fault-tolerant control using smart actuators and time-triggered communication [Ph.D. dissertation], Department of Automatic Control and Systems Engineering, Sheffield University, Sheffield, UK, 2002.

[13] C. Kambhampati, R. J. Patton, and F. J. Uppal, "Reconfiguration in networked control systems: fault tolerant control and plugand-play," in Proceedings of the 6th IFAC Symposium on Fault Detection, Supervision and Safety of Technical Processes, pp. 151156, Tsinghua University, Beijing, China, 2006.

[14] J. D. Bošković and R. K. Mehra, "Failure detection, identification and reconfiguration in flight control," in Fault Diagnosis and Fault Tolerance for Mechatronic Systems: Recent Advances, vol. 1, pp. 129-167, Springer, Berlin, Germany, 2003.

[15] K. W. Iliff and K.-S. C. Wang, "Flight-determined subsonic longitudinal stability and control derivatives of the F-18 High Angle of Attack Research Vehicle (HARV) with thrust vectoring," Tech. Rep. NASA/TP-97-206539, 1997.

[16] J. A. Richard, J. M. Buffington, A. G. Sparks, and S. S. Banda, Robust Multivariable Flight Control, Springer, London, UK, 1994.

[17] J. M. Buffington and D. F. Enns, "Lyapunov stability analysis of daisy chain control allocation," Journal of Guidance, Control, and Dynamics, vol. 19, no. 6, pp. 1226-1230, 1996.

[18] D. Enns, D. Bugajski, R. Hendrick, and G. Stein, "Dynamic inversion: an evolving methodology for flight control design," International Journal of Control, vol. 59, no. 1, pp. 71-91, 1994.

[19] "Military Standard Flying Qualities of Piloted Aircraft," MilSTD-1797A, 1990. 


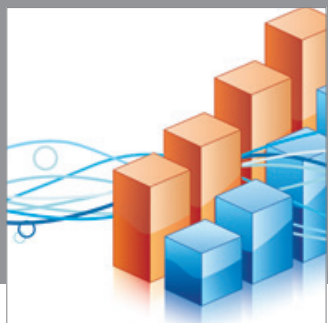

Advances in

Operations Research

mansans

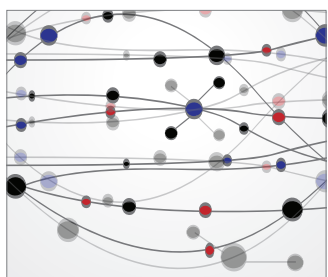

The Scientific World Journal
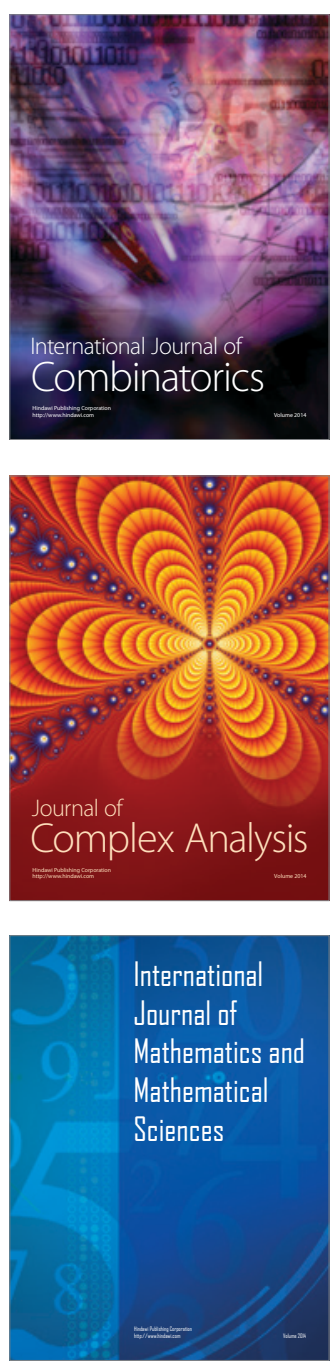
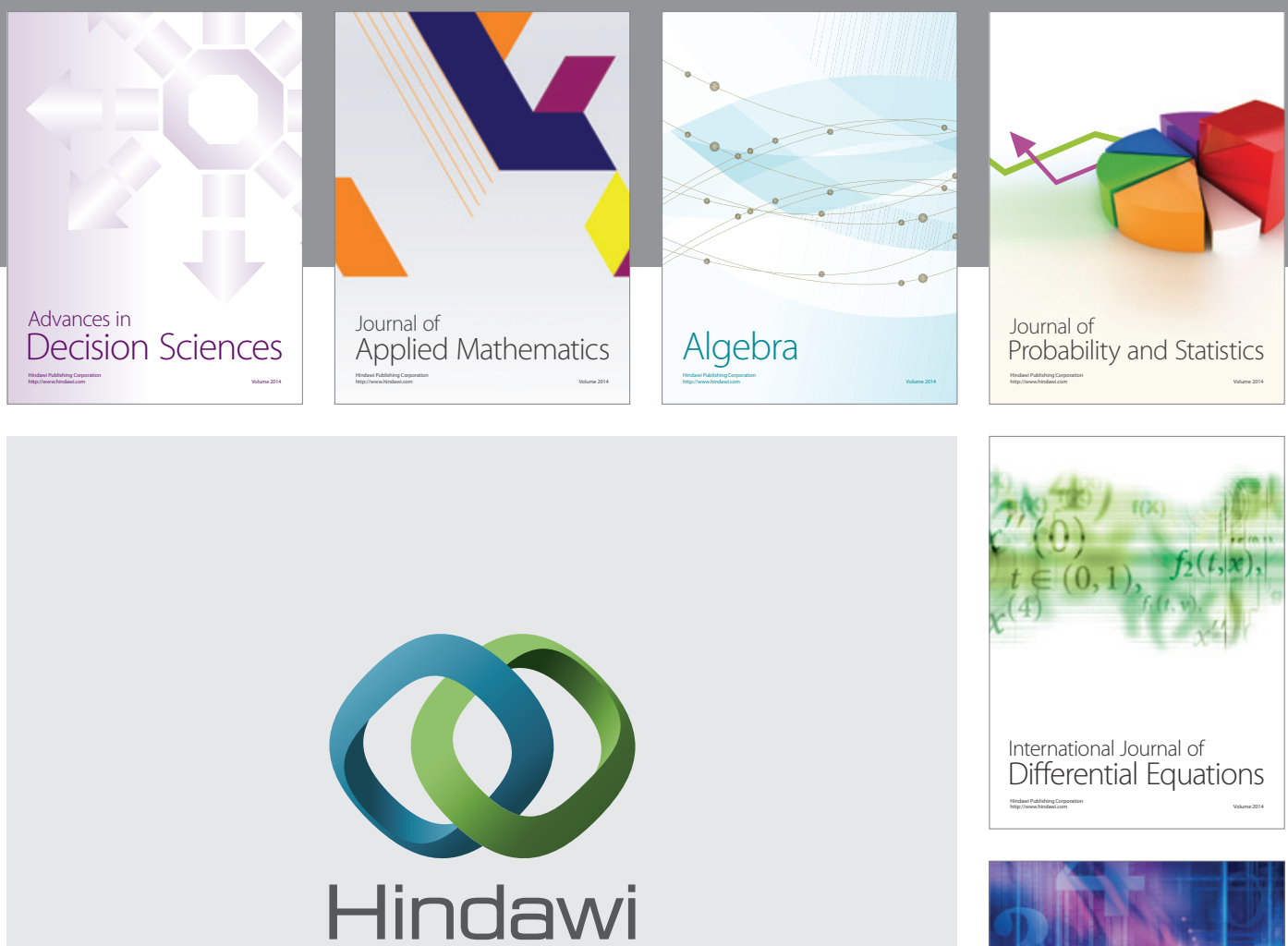

Submit your manuscripts at http://www.hindawi.com
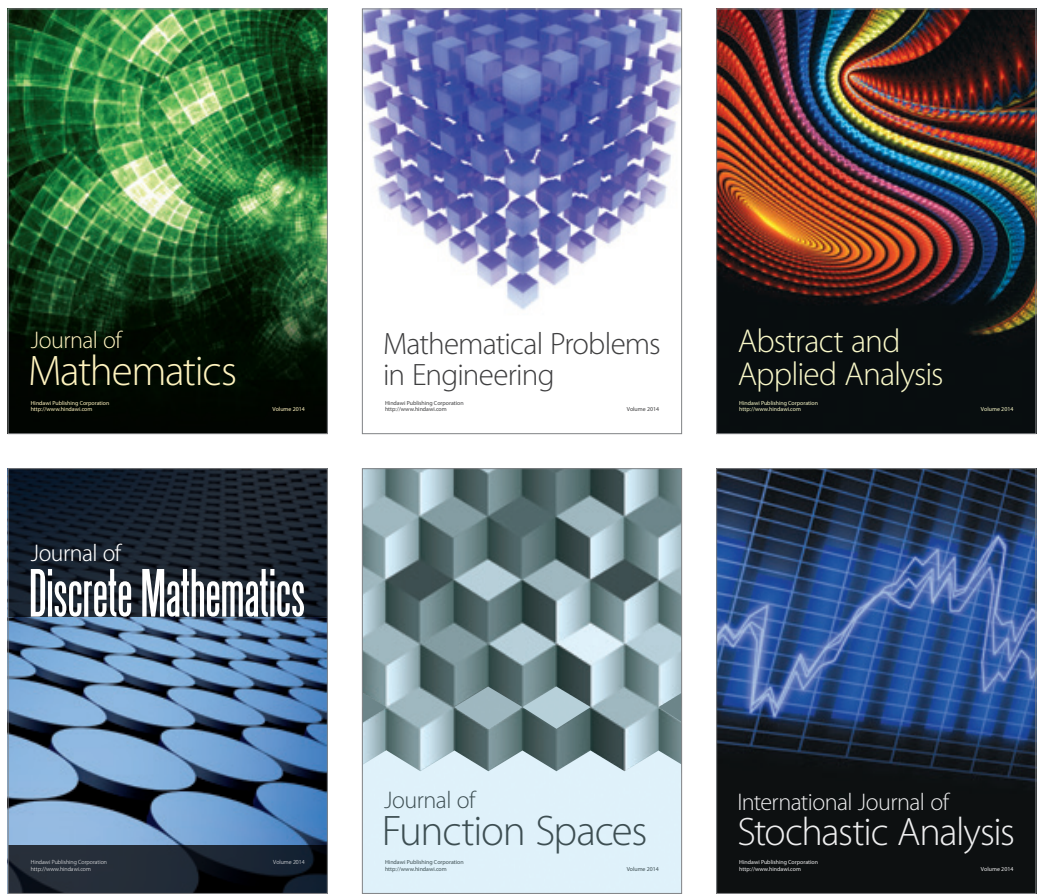

Journal of

Function Spaces

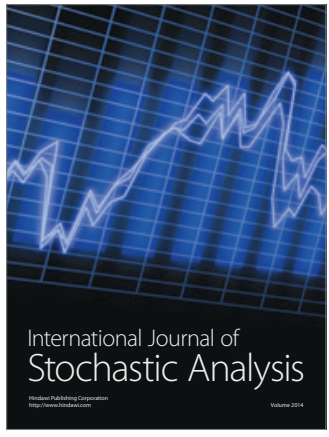

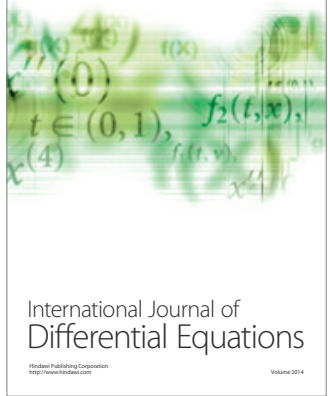
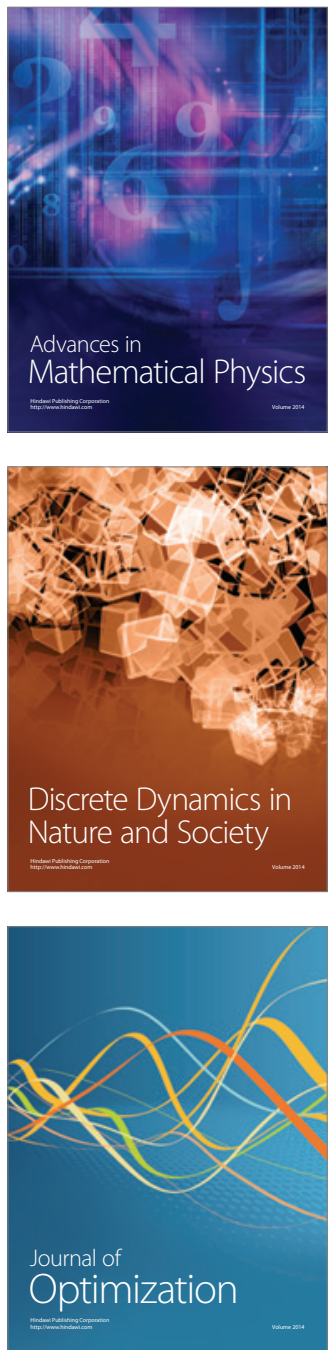